\title{
Source of Energetic Protons in the 2014 September 1 Sustained Gamma-ray Emission Event
}

\author{
N. Gopalswamy ${ }^{1}(D)$ P. Mäkelä2 $\cdot S$. Yashiro ${ }^{2}$. \\ S. Akiyama ${ }^{2}$ H. Xie ${ }^{2} \cdot$ N. Thakur ${ }^{2}$
}

Received: 11 October 2019 / Accepted: 23 January 2020 / Published online: 4 February 2020

(C) The Author(s) 2020

\begin{abstract}
We report on the source of $>300 \mathrm{MeV}$ protons during the SOL2014-09-01 sustained gamma-ray emission (SGRE) event based on multi-wavelength data from a wide array of space- and ground-based instruments. Based on the eruption geometry we provide concrete explanation for the spatially and temporally extended $\gamma$-ray emission from the eruption. We show that the associated flux rope is of low inclination (roughly oriented in the east-west direction), which enables the associated shock to extend to the frontside. We compare the centroid of the SGRE source with the location of the flux rope's leg to infer that the high-energy protons must be precipitating between the flux rope leg and the shock front. The durations of the SOL2014-09-01 SGRE event and the type II radio burst agree with the linear relationship between these parameters obtained for other SGRE events with duration $\geq 3 \mathrm{hrs}$. The fluence spectrum of the SEP event is very hard, indicating the presence of high-energy $(\mathrm{GeV})$ particles in this event. This is further confirmed by the presence of an energetic coronal mass ejection with a speed $>2000 \mathrm{~km} \mathrm{~s}^{-1}$, similar to those in ground level enhancement (GLE) events. The type II radio burst had emission components from metric to kilometric wavelengths as in events associated with GLE events. All these factors indicate that the high-energy particles from the shock were in sufficient numbers needed for the production of $\gamma$-rays via neutral pion decay.
\end{abstract}

Keywords Coronal mass ejections · Flares · Flux rope $\cdot$ Shock $\cdot$ Magnetic cloud, gamma-rays · Type II radio burst $\cdot$ Solar energetic particle event

\section{Introduction}

Gamma-ray events temporally extended beyond the impulsive phase of solar flares were first reported by Forrest et al. (1985) using the Solar Maximum Mission's Gamma Ray Spectrometer (SMM/GRS) data. During the 1982 June 3 event, the $\gamma$-ray emission extended

\footnotetext{
$凶$ N. Gopalswamy

nat.gopalswamy@nasa.gov

1 NASA Goddard Space Flight Center, Greenbelt, MD, USA

2 The Catholic University of America, Washington, DC, USA
} 
beyond the flare impulsive phase by $\sim 20$ min and was recognized as the neutral-pion-decay continuum based on different spectra during the impulsive and late phases. The Gamma-ray burst experiment (PHEBUS) on board the GRANAT mission also observed extended-phase emission at energies $>10 \mathrm{MeV}$ (Talon et al., 1993; Vilmer et al., 2003). Using data from Gamma-1 telescope, Akimov et al. (1991) reported on the 1991 June 15 event with the extended phase $\gamma$-ray emission lasting for more than $2 \mathrm{hr}$. Based on data from the Energetic Gamma Ray Experiment Telescope (EGRET) on board the Compton Gamma Ray Observatory (CGRO), Kanbach et al. (1993) reported on another event with a duration exceeding $8 \mathrm{hr}$. After the advent of the Fermi Large Area Telescope (Fermi/LAT, Atwood et al., 2009) it has become clear that such extended-duration $\gamma$-ray events are rather common (Ackermann et al., 2014; Share et al., 2018; Winter et al., 2018). To underline the fact that $\gamma$-ray photons are emitted long after the associated flares, the emission is now referred to as sustained $\gamma$-ray emission (SGRE) (see e.g., Plotnikov, Rouillard, and Share, 2017; Klein et al., 2018; Gopalswamy et al., 2018a; Kahler, Cliver, and Kazachenko, 2018). As pointed out by Ryan (2000), the definition of the long-duration $\gamma$-ray events has been imprecise: the term "longduration gamma-ray flare (LDGRF)" refers to the $\gamma$-rays as flare, although the flare is gone long before the end of the $\gamma$-ray events.

During SGRE events, the photon spectrum extends to energies $>1 \mathrm{GeV}$ and has a peak around $70 \mathrm{MeV}$, characteristic of $\gamma$-rays from neutral pion decay. It was already recognized that the extended $\gamma$-ray emission is related to the process accelerating SEPs that is distinct from the impulsive-phase acceleration (Forrest et al., 1985). The definite evidence that large SEP events are produced by shocks driven by coronal mass ejections (CMEs) was first reported by Kahler, Hildner, and Van Hollebeke (1978), soon after the discovery of CMEs in white light (Tousey, 1973) (see Reames, 1999 for a review). Therefore, the idea of shocks supplying the necessary $>300 \mathrm{MeV}$ protons has also been proposed soon after the discovery of the long duration $\gamma$-ray flares (Murphy, Dermer, and Ramaty, 1987; Ramaty, Murphy, and Dermer, 1987). Nevertheless, the idea of impulsivephase particles trapped in long loops and precipitating slowly to the photosphere continues to be pursued (e.g., Hudson, 2018; Grechnev et al., 2018; De Nolfo et al., 2019a; De Nolfo et al., 2019b).

Citing the presence of metric type II burst, Akimov et al. (1991) suggested that the protons responsible for the $\gamma$-ray emission should have been shock-accelerated. The $2.223 \mathrm{MeV}$ $\gamma$-ray line (GRL) emission observed by SMM/GRS from a backside eruption on 1989 March 29 located $\sim 10^{\circ}$ behind the limb (Vestrand and Forrest, 1993; Cliver, Kahler, and Vestrand, 1993) was shown to be consistent with a shock source for the energetic protons. The $2.223 \mathrm{MeV}$ line is produced deep in the chromosphere, so it would not reach the observer on the Sun-Earth line from behind the limb. However, particles accelerated at the front of the associated shock can readily supply particles precipitating on the frontside of the Sun to produce the line emission (Cliver, Kahler, and Vestrand, 1993; Vestrand and Forrest, 1993). Cliver, Kahler, and Vestrand (1993) provided details of the CME that was driving the shock and the spatial extent of the CME/shock was consistent with the spatially extended GRL emission. The shock acceleration was further corroborated by the presence of a metric type II radio burst, which is indicative of the shock near the Sun. Cliver, Kahler, and Vestrand (1993) were cautious to state that it was an open question whether shock mechanism applicable to GRL emission would also apply to SGREs. The observation of SGRE from three backside events by Fermi/LAT provided definite evidence that the time-extended emission is also spatially extended (Pesce-Rollins et al., 2015a; Pesce-Rollins et al., 2015b; Plotnikov, Rouillard, and Share, 2017; Ackermann et al., 2017; Jin et al., 2018). In particular, the SOL2014-09-01 (Sep14, for short) event was extraordinary in that the eruption was $\sim 40^{\circ}$ behind the limb, yet SGRE was observed by Fermi/LAT 
suggesting that the SGRE emission is truly spatially extended and must be due to a CMEdriven shock.

Share et al. (2018) noted that most of the SGRE events are associated with a fast CME $\left(>800 \mathrm{~km} \mathrm{~s}^{-1}\right)$ and a type II burst at decameter-hectometric (DH) wavelengths. One of the most recent revelations is that the duration of SGRE events is linearly related to that of DH type II bursts and inversely related to the ending frequency of the type II bursts (Gopalswamy et al., 2018a). These observations provide the physical basis for the time-extended nature of SGRE events: life time of such SGRE events is determined by the duration over which the underlying CME-driven shock efficiently accelerates $>300 \mathrm{MeV}$ protons that propagate to the solar surface and produce $\gamma$-ray emission via the decay of neutral pions. Since there is a one-to-one correspondence between large SEP events and DH type II bursts extending from metric to kilometric wavelengths (Gopalswamy et al., 2008), it is highly likely that the same shock is responsible for $>300 \mathrm{MeV}$ protons (for SGRE) and $\sim 10 \mathrm{keV}$ electrons (for DH type II burst). It must be noted that the shock continues to accelerate particles to lower energies (e.g., energetic storm particle events are often observed after the end of SGRE) and weak/fragmented type II bursts are observed until the shock arrives at the observing spacecraft such as Wind and STEREO.

Several papers have already appeared dealing with various aspects of the Sep14 event, but here we focus on the source of $>300 \mathrm{MeV}$ protons that provides a concrete explanation for the spatially and temporally extended $\gamma$-ray emission from a solar eruption. We show that the SGRE duration and type II burst duration are consistent with the linear relationship found by Gopalswamy et al. (2018a). Furthermore, we exploit the two-view observations Solar Terrestrial Relations Observatory (STEREO, Kaiser et al., 2008) and the Solar and Heliospheric Observatory (SOHO, Domingo, Fleck, and Poland, 1995) to derive the flare, flux-rope, and shock structures in the eruption that are consistent with the idea of shock particles precipitating on the frontside of the Sun to produce the observed SGRE event.

\section{Data Description}

Sep14 is the most intense and longest-lasting among the three behind-the-limb SGRE events observed by Fermi/LAT (Pesce-Rollins et al., 2015a, 2015b; Share et al., 2018; Ackermann et al., 2017; Plotnikov, Rouillard, and Share, 2017; Jin et al., 2018; Hudson, 2018; Grechnev et al., 2018). Plotnikov, Rouillard, and Share (2017), Ackermann et al. (2017), and Share et al. (2018) described the full time evolution of the SGRE flux. Share et al. (2018) showed that the SGRE event was distinct from the impulsive-phase emission. Details of the eruption region and the impulsive-phase radio and X-ray emissions have been already described in these papers. Here we describe additional data that help fully understand the eruptive event and the SEP that led to the SGRE. Ground-based radio instruments observed radio bursts of various types: type II, type III, and type IV continuum (Carley et al., 2017). The radio bursts also continued into the interplanetary (IP) medium as observed by the Radio and Plasma Wave Experiment (WAVES, Bougeret et al., 1995) on board the Wind and STEREO (SWAVES, Bougeret et al., 2008) spacecraft. We make use of the fact that the type II radio burst was observed by SWAVES without obstruction, so we obtain the full evolution of the burst in the radio dynamic spectrum. The early phase of the shock has already been described in previous papers (Plotnikov, Rouillard, and Share, 2017; Jin et al., 2018; Grechnev et al., 2018). Here we provide information on the shock and CME as observed by the Large Angle and Spectrometric Coronagraph (LASCO, Brueckner et al., 1995) on board SOHO and the Sun Earth Connection Coronal and Heliospheric Investigation 

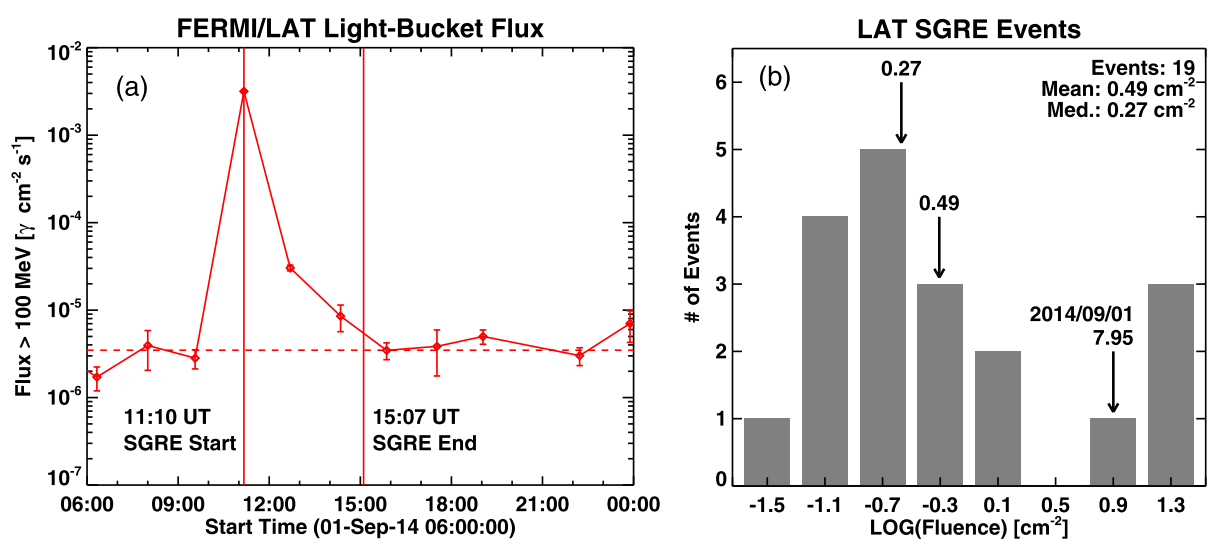

Figure 1 Time profile of the Sep14 SGRE event (a) and its fluence compared with the $>3$ hr SGRE events observed by Fermi/LAT (b). The duration over which the fluence was computed is shown in (a). The horizontal dashed line in (a) represents the celestial and solar quiescent backgrounds within a 10-degree circle around the Sun. The Sep14 event is the fourth largest among the 19 SGRE events that have durations exceeding $3 \mathrm{hr}$.

(SECCHI, Howard et al., 2008) on board STEREO. More details on the DH type II burst can be found in https://cdaw.gsfc.nasa.gov/CME_list/radio/waves_type2.html. CME properties measured in the sky plane are available online in the SOHO/LASCO CME catalog (https://cdaw.gsfc.nasa.gov, Yashiro et al., 2004; Gopalswamy et al., 2009a). To get the three-dimensional speeds, we fit a flux rope to the CME observed in coronagraph and EUV images using the graduated cylindrical shell (GCS) model (Thernisien, 2011) and a spheroid to the shock ahead of the CME (Olmedo et al., 2013; Hess and Zhang, 2014; Mäkelä et al., 2015; Xie et al., 2017; Gopalswamy et al., 2018b). The presence of $>300 \mathrm{MeV}$ protons is a critical requirement for the SGRE events. We use GOES $>100 \mathrm{MeV}$ proton data available from NOAA's Space Weather Prediction Center (SWPC) as a proxy to the $>300 \mathrm{MeV}$ protons. For particles arriving at STEREO spacecraft, we use data from the High Energy Telescope (HET) and Low Energy Telescope (LET) of the In Situ Measurements of Particles and CME Transients (IMPACT, von Rosenvinge et al., 2008). The highest-energy channel of the STEREO particle detectors is $\sim 100 \mathrm{MeV}$. In order to get $>100 \mathrm{MeV}$ integral fluxes similar to GOES, we extrapolate the proton spectrum to $\sim 1 \mathrm{GeV}$ and assume that there is little contribution from particles at energies $>1 \mathrm{GeV}$. We also obtained the fluence spectrum of the event from STEREO and GOES data.

\section{Analysis and Results}

We define the SGRE duration as the interval from the end of the impulsive phase as marked by the GOES soft X-ray peak and the midpoint between the last signal data point above the background level and the one after that (Gopalswamy et al., 2018a). Note that Fermi/LAT observes the Sun only intermittently due to the continuously changing pointing direction of the instrument. The SGRE peak occurred after the impulsive phase of the flare as revealed by the hard X-ray emission observed by the High Energy Neutron Detector (HEND) of the Gamma-ray Spectrometer onboard the Mars Odyssey mission (see Grechnev et al., 2018 for details). Therefore, we estimate the duration of the event from the SGRE peak at 11:10 UT to 15:07 UT as 3.92 $\pm 0.76 \mathrm{hr}$. Figure 1 shows the SGRE time profile and the fluence compared 
Figure 2 Locations of STEREO B (B), Mercury, and Mars with respect to Earth at the time of the Sep14 event. The CME direction (E127) is shown. The CME was front-sided to STEREO-B, Mercury MESSENGER, and Mars Odyssey. The line above the east limb of the Sun from Earth view is marked. For STEREO-B, the eruption is a western event; for MESSENGER and Mars Odyssey it is an eastern event.

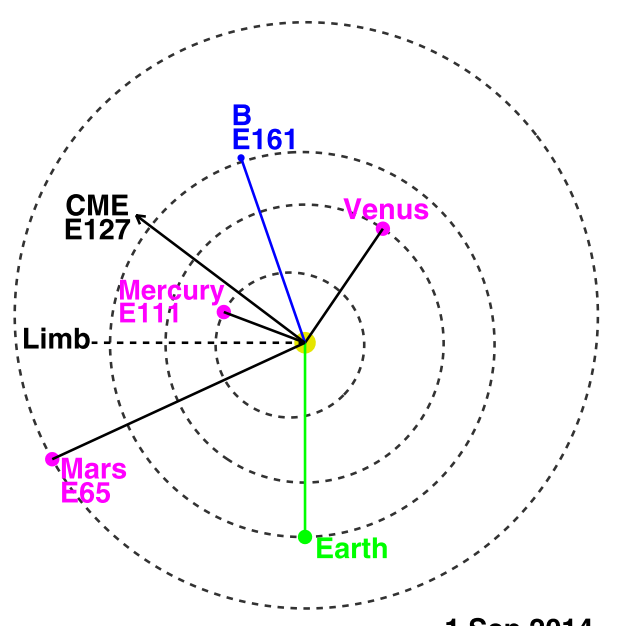

1 Sep 2014

with those of other SGRE events of duration $>3 \mathrm{hr}$. The Sep14 event had the fourth largest fluence $\left(7.94 \mathrm{~cm}^{-2}\right)$. Some of the $\gamma$-ray photons would not have reached Fermi/LAT, so the true fluence of the Sep14 event might have been higher. The three events with higher fluence are: 2012 March $7\left(24.1 \mathrm{~cm}^{-2}\right)$, 2014 February $25\left(14.9 \mathrm{~cm}^{-2}\right)$, and 2017 September 10 $\left(13.9 \mathrm{~cm}^{-2}\right)$. The fluence values reported here are slightly different from those in Winter et al. (2018) because their start times are different from ours. For example, in the Sep14 event, we use the peak time of the soft X-ray emission (11:10 UT) as the starting time, whereas Winter et al. (2018) use 11:02 UT, which is in the impulsive phase.

\subsection{The Solar Source}

The Sep14 SGRE event originated from an active region located $\sim 37^{\circ}$ behind the east limb (N14E127-see Ackermann et al., 2017; Plotnikov, Rouillard, and Share, 2017; Jin et al., 2018; Grechnev et al., 2018). Fortunately, there were three space observatories in which the source was observed as a frontside event (see Figure 2). STEREO Behind (STB) spacecraft was located at E161, so the solar source location is N14W34 in that view. STB's Extreme Ultra-violet Imager (EUVI) imaged the eruption region including the coronal dimming and post-eruption arcade (PEA), while COR1 and COR2 imaged the CME from the inner corona to the IP medium. STB was also well connected to the SEP event. The hard X-ray emission from the flare was observed by HEND onboard the Mars Odyssey mission (see Grechnev et al., 2018 for details). Mars Odyssey was located at a longitude of E62, so the eruption was a disk event ( $\sim$ E65) in the spacecraft's view. Mercury MESSENGER was at a longitude of E110, so the eruption was a disk-center (E17) event in its view. The Solar Assembly for X-rays (SAX), which is the Sun-pointed detector of the X-Ray Spectrometer (XRS) on board MESSENGER detected the soft X-ray flare (see Share et al., 2018 for details). The SGRE source location was estimated as N41E90 by Ackermann et al. (2017). However, the source location has been revised and the new location is closer to the latitude of the eruption site (M. Pesce-Rollins, private communication). In any case, the SGRE source longitude is W71 in STB view, which is about $37^{\circ}$ west of the active region.

The STB/EUVI images in Figure 3 show the region of interest before and after the eruption. The eruption resulted in a PEA oriented along a NE-SW line and a twin dimming (D1, D2). When the active region rotated onto the disk a few days later (September 5 onwards), it 

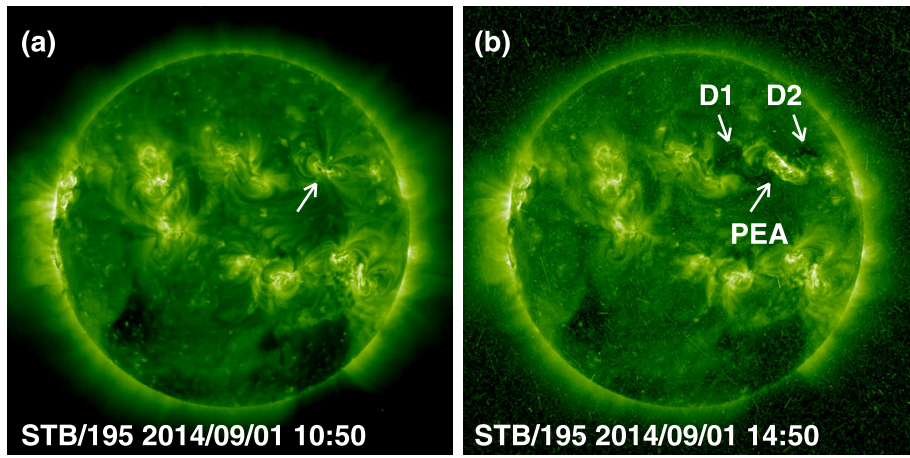

Figure 3 An overview of the Sep14 eruption as seen in STB/EUVI images taken just before the eruption (b) and in the post-eruption phase (b). In (a), the arrow points to the eruption region. In (b), the two dimming regions D1, D2 and the post-eruption arcade (PEA) are marked. The "snowstorm" in the image in (b) is due to energetic particles from the eruption hitting the STB detectors.
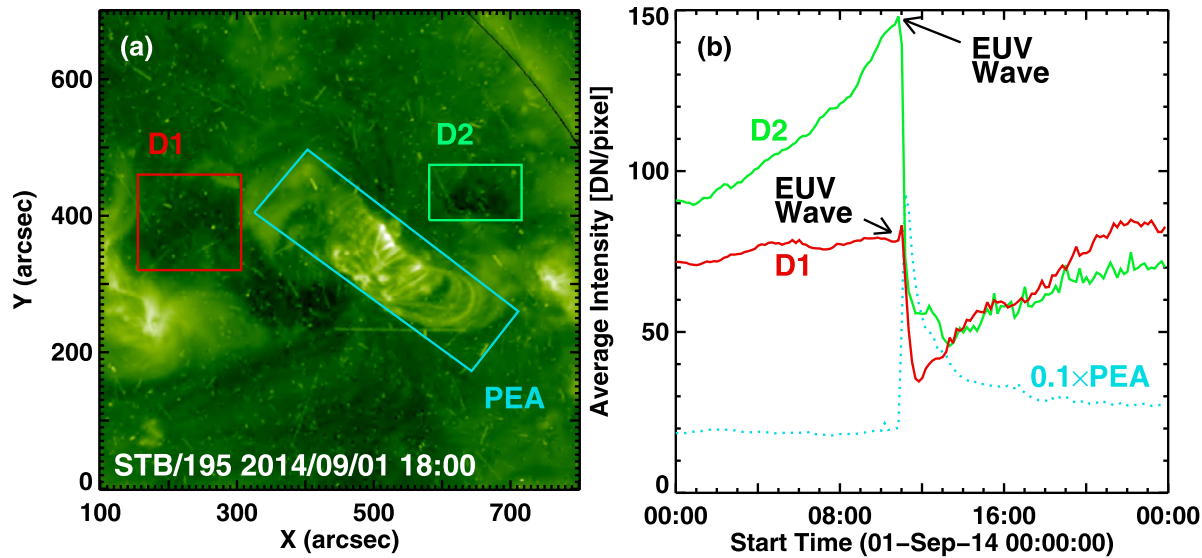

Figure 4 The core dimming regions D1, D2, and the PEA of the Sep14 eruption (a) and the time evolution of the intensities (b) in those regions. The small spike in D1 and D2 correspond to the arrival of the EUV wave above these regions. The intensity of the PEA (blue dotted line) is equivalent to the soft X-ray flare. There was additional dimming to the southwest of D1 caused by the removal of an overlying structure due to the eruption. This dimming did not recover as D1 and D2 did. The concerned active region rotated on to the disk as NOAA AR 12158. The region produced an eruption on 2014 September 10. The PEA at that time was more east-west than in the present event. The box D2 was fixed while the Sun was rotating underneath. Some bright structures in the outer part of the active region rotated into the box, increasing the signal slowly (over $\sim 12$ hours), unrelated to the eruption.

was found that the leading polarity was positive. The dimming regions D1 and D2 had negative and positive polarities, respectively. The inclination of the arcade was $\sim-34^{\circ}$, while the line joining the dimming regions was almost horizontal $\left(-12^{\circ}\right.$ inclination). Figure 4 shows the evolution of the EUV intensity in the dimming regions D1 and D2 and the PEA. The spikes in the dimming region plots correspond to the EUV wave dome passing these regions before the dimming starts. D1 and D2 are also referred to as core dimming and thought to be the locations where the CME flux rope footpoints are rooted (Webb et al., 2000; Dissauer et al., 2018; Gopalswamy et al., 2018b). The deepest dimming occurred slightly 

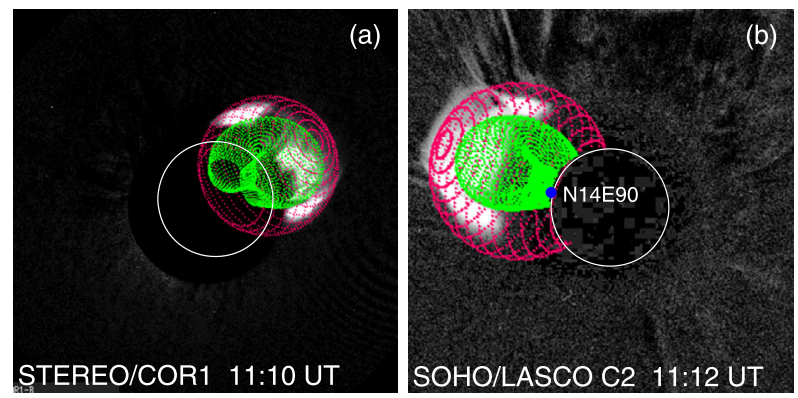

Figure 5 Flux rope (green) and shock (pink) obtained from the forward-modeling fit to the CME from STEREO and SOHO observations. The flux rope and shock are shown superposed on STB/COR1 image at 11:10 UT (a) and SOHO/LASCO/C2 image at 11:12 UT. The blue dot on the east limb is the SGRE centroid obtained from Fermi/LAT. The location corresponds to the outskirts of the western leg of the flux rope within the shock sheath as seen in STB view. The SGRE flux reached its peak value around the time of these images. The shock and flux rope leading edges are at 3.2 and 2.8 Rs, respectively. The GCS fit also gives the ratio of the flux rope radius to the heliocentric distance of the flux rope nose as 0.24 .

before $12 \mathrm{UT}$, but there was a small reversal in D2. The recoveries were similar in D1 and D2 for several hours. The EUV intensity of the PEA peaks around 11:10 UT, coincident with the soft X-ray peak observed by SAX/XRS onboard MESSENGER (Share et al., 2018). The equivalent soft X-ray size was estimated using the similarity (Nitta et al., 2013; Chertok, Belov, and Grechnev, 2015) between EUV and X-ray time variations as X2.4 (Ackermann et al., 2017).

\subsection{The CME and Shock}

Mass motion from the eruption region was observed in SDO images starting at 10:58 UT as reported by Grechnev et al. (2018), with a leading-edge height at 1.35 Rs at 11:00:01 UT. The CME was also observed by GOES SXI at 11:01:15 UT with the leading edge at a height of 1.7 Rs (Carley et al., 2017). Both observations were in sky-plane projection. In order to get the three-dimensional speed of the CME and shock, we used STEREO and SOHO observations to fit a flux rope to the CME and a spheroid to the leading shock dome using the GCS model for the flux rope (Thernisien, 2011) and the spheroidal model for the shock (Olmedo et al., 2013). The flux rope-shock model has been applied successfully to track the leading edges of CME events (Hess and Zhang, 2014; Mäkelä et al., 2015; Xie et al., 2017).

Figure 5 shows the shock and flux rope fits to STEREO and SOHO coronagraph images at 11:10 UT and 11:12 UT, respectively. The direction of propagation of the flux rope nose is along N16E117, which corresponds to a slight westward deflection of the flux rope compared to the radial direction (N14E127). The deflection brings the nose closer to the limb ( $\sim 27^{\circ}$ behind the limb in Earth view). The tilt angle of the flux rope is $-12^{\circ}$, which is consistent with the east-west placement of the core dimming regions D1 and D2 (see Figure 4) and different from the tilt of the PEA $\left(\sim-34^{\circ}\right)$. The flux rope's face-on and edge-on half widths are $38^{\circ}$ and $18^{\circ}$, respectively. Recall that the angular distance from the source region to the west limb in STB view is $\sim 37^{\circ}$. This means the western edge of the flux rope extends to the frontside of the Sun. The shock, which is more extended than the flux rope, clearly extends to the frontside and is consistent with the EUV wave crossing the STB west limb and propagating to the frontside (Plotnikov, Rouillard, and Share, 2017; Jin et al., 2018). In 

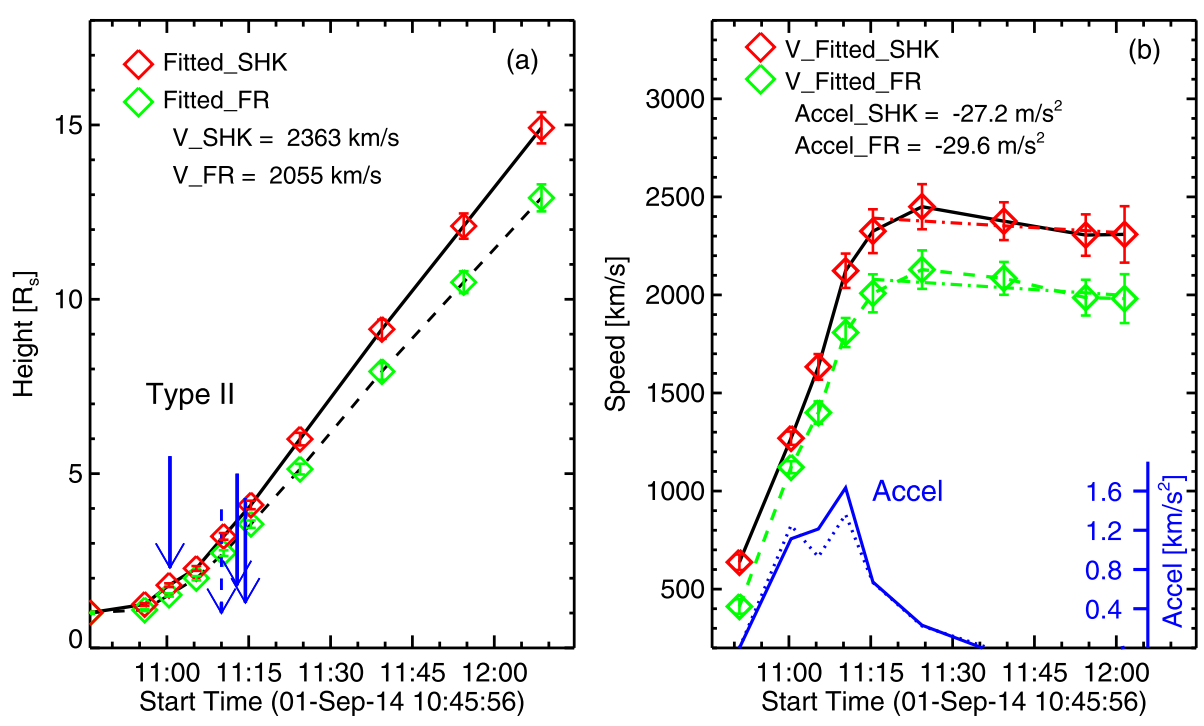

Figure 6 Kinematics of the Sep14 flux rope (green data points and dashed line) and shock (red data points and solid line) based on the GCS and spheroidal models. (a) height-time plot of the shock and flux rope. The onset times of metric (solid arrows) and DH (dashed arrow) type II bursts are marked. (b) The evolution of shock and CME speeds and the acceleration of shock (solid blue line) and flux rope (dashed blue line) derived from the height-time data points. A peak shock acceleration was $1.6 \mathrm{~km} \mathrm{~s}^{-2}$ attained at 11:10 UT. The dot-dashed lines are best-fit lines to the decreasing speeds of the flux rope and shock.

the case of high-inclination flux ropes, the east-west extent would be smaller and a source at $40^{\circ}$ behind the limb may not produce a $\gamma$-ray event on the Earth-facing disk. The inclination difference was previously invoked by Gopalswamy et al. (2015a) to explain why the 2014 January 6 SEP event was a GLE event (low-inclination flux rope), while the 2012 May 27 SEP was not (high-inclination flux rope) even though the eruption longitudes were similar behind the west limb.

Figure 6 shows the height, speed, and acceleration of the shock and flux rope as a function of time within the STB/COR2 field of view (FOV). The shock and the flux rope have average speeds exceeding $2000 \mathrm{~km} \mathrm{~s}^{-1}$. The shock speed attains a peak value of $\sim 2450 \mathrm{~km} \mathrm{~s}^{-1}$ at 11:25 UT and then decrease slowly due to the aerodynamic drag but remains above $2000 \mathrm{~km} \mathrm{~s}^{-1}$ within COR2 FOV. The speeds and initial accelerations are typical of CMEs producing GLEs in SEP events. For comparison, the CME speed is in between that of the two GLE events of solar cycle 24: 2012 May 17 ( $2000 \mathrm{~km} \mathrm{~s}^{-1}$; Gopalswamy et al., 2013a) and 2017 September $10\left(\sim 3400 \mathrm{~km} \mathrm{~s}^{-1}\right.$; Gopalswamy et al., 2018c). The initial acceleration $\left(1.66 \mathrm{~km} \mathrm{~s}^{-2}\right)$ is similar to that of the 2012 May 17 event $\left(1.77 \mathrm{~km} \mathrm{~s}^{-2}\right)$, but much smaller than that of the 2017 September 10 event $\left(9.1 \mathrm{~km} \mathrm{~s}^{-2}\right)$. The CME and shock kinematics are thus consistent with an energetic eruption capable of accelerating particles to $\mathrm{GeV}$ energies.

\subsection{Radio Bursts}

The Sep14 SGRE event was accompanied by complex radio emission from microwave to kilometer wavelengths indicating the presence of accelerated electrons. Details of the metric and microwave emission have been reported in Figure 1 of Carley et al. (2017) and in Figure 9 of Grechnev et al. (2018). Here we provide a comprehensive description including the 


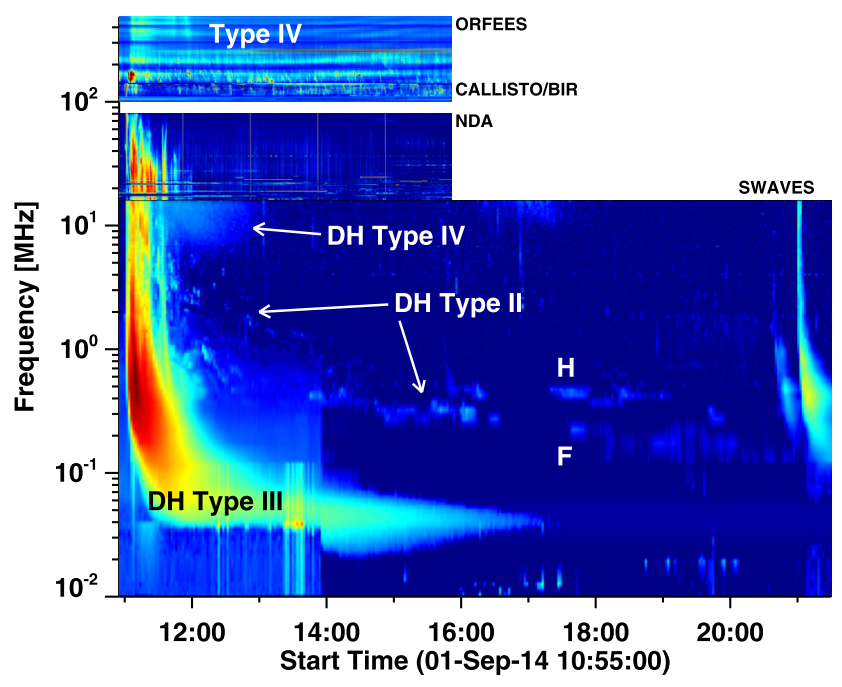

Figure 7 Radio emissions associated with the Sep14 SGRE event shown in the form of a composite dynamic spectrum. Observations at frequencies $>100 \mathrm{MHz}$ are from the Orfées and CALLISTO instruments. Nancay Decametric Array (NDA) observes in the range 10-88 MHz at both polarizations, but here we have shown the total intensity. The sharp start of radio emission at $\sim 180 \mathrm{MHz}$ is most likely due to the occultation of plasma levels $>90 \mathrm{MHz}$ (the radio emission observed at the limb is typically at harmonic). Decameter-hectometric (DH) Type II, type III and type IV emissions are marked. The bright emission in the NDA band continues as the long duration type IV. The DH type II has fundamental-harmonic (F-H) structure.

IP components. The IP components are particularly important, because they indicate continued particle acceleration required for the SGRE event. Figure 7 shows a composite dynamic spectrum in the range $500 \mathrm{MHz}$ to $10 \mathrm{kHz}$. The data above $100 \mathrm{MHz}$ are from the Orfées and CALLISTO spectrographs. The Nancay Decametric Array (NDA) data were used in the range 10-88 MHz. At frequencies below $16 \mathrm{MHz}$, we have used the SWAVES data from STB. The type IV emission above $\sim 200 \mathrm{MHz}$ was interpreted as moving type IV burst by Carley et al. (2017) due to $\sim \mathrm{MeV}$ electrons trapped in the CME flux rope structure. These frequencies are not occulted because the flux rope (and hence the moving type IV source) is already above the limb at the start of the emission $(\sim 11: 01 \mathrm{UT})$ as evident from the height of the soft X-ray transient ( $\sim 1.69$ Rs at 11:01:15 UT). Intense metric radio emission starts at $<180 \mathrm{MHz}$. The emission consists of a series of type III bursts, three episodes of type II bursts, and a flare continuum.

In the DH domain, the eruption was accompanied by type III, type II, and type IV bursts. The DH type III bursts were a continuation of type III bursts in the NDA frequency range and lasted until $\sim 11: 25$ UT. The DH type II burst crosses the upper edge of the dynamic spectrum $(\sim 16 \mathrm{MHz})$ around $\sim 11: 10 \mathrm{UT}$ and continues as fragmented emission until $\sim$ 20:00 UT with an ending frequency below $200 \mathrm{kHz}$. However, the burst has a clear break during 16:15 to 17:30 UT. Type III bursts typically mark electron acceleration in the impulsive phase of the associated flare and propagation along open field lines, while the type IV indicates electrons trapped in tall flare structures, i.e., PEAs (Gopalswamy, 2011). Type IV bursts at frequencies below $10 \mathrm{MHz}$ are very rare and are associated with CMEs with very high average speed $\left(\sim 1500 \mathrm{~km} \mathrm{~s}^{-1}\right)$. The low-frequency edge of the type IV burst descends to lower frequencies, reaching a minimum of $\sim 7 \mathrm{MHz}$ at $12 \mathrm{UT}$ and then increases, reaching the upper edge of the SWAVES frequency range an hour later. The type II 
Figure 8 Composite dynamic spectrum showing nonthermal radio emission at frequencies $>10 \mathrm{MHz}$. The plus symbols denote the starting time/frequency of the three type II episodes with the fundamental and harmonic components denoted by F1, F2, F3 and H1, $\mathrm{H} 2, \mathrm{H} 3$. The horizontal dashed line at $180 \mathrm{MHz}$ denotes the high-frequency cutoff of the plasma emissions. The type IV burst at frequencies above $200 \mathrm{MHz}$ is gyrosynchrotron emission from the CME flux rope.

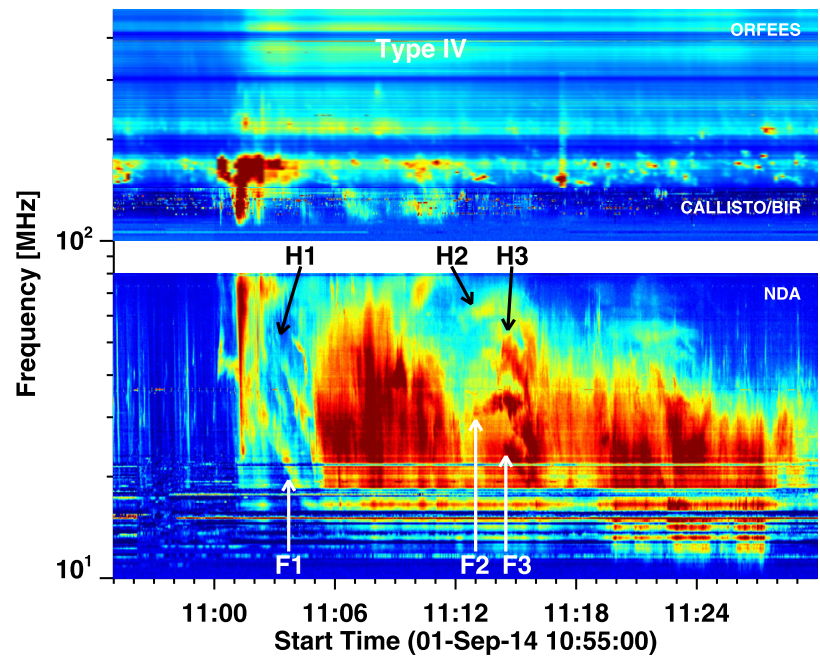

Table 1 Type II burst episodes from NDA during the Sep14 eruption.

\begin{tabular}{lllll}
\hline Episode & Starting time (UT) & Starting frequency $(\mathrm{MHz})$ & Harmonic present? & Ending time (UT) \\
\hline 1 & $11: 00: 34$ & 45.1 & Yes & $11: 05$ \\
2 & $11: 12: 53$ & 32.9 & Yes & $11: 16$ \\
3 & $11: 14: 24$ & 25.6 & Yes & $11: 16$ \\
\hline
\end{tabular}

burst is at the descending edge of the type IV burst, which is typically the case when type II and type IV bursts occur together. While the type II and type III bursts are also observed by Wind/WAVES, the type IV burst was not because of its directivity (Gopalswamy et al., 2016a). It must be noted that the presence of type II emission from metric to kilometric domains is a characteristic of GLE events indicating the presence of $\sim \mathrm{GeV}$ protons.

Carley et al. (2017) fitted a gyrosynchrotron spectrum to the higher-frequency radio observations that peaked at $\sim 1000 \mathrm{MHz}$ and inferred a magnetic field strength of $4.4 \mathrm{G}$ at a height of $\sim 1.3 \mathrm{Rs}$. This value is consistent with the range of axial field strengths (10 to $100 \mathrm{mG}$ at $10 \mathrm{Rs}$ ) of coronal flux ropes obtained by Gopalswamy et al. (2018d). A magnetic field of $4.4 \mathrm{G}$ at $1.3 \mathrm{Rs}$ corresponds to an axial field strength of $\sim 75 \mathrm{mG}$ at $10 \mathrm{Rs}$, assuming self-similar expansion of the flux rope. This is above the average of the distribution $(52 \mathrm{mG})$ because the eruption is very energetic: higher total reconnected flux in the eruption region results in higher axial field strength and larger CME kinetic energy (Gopalswamy et al., 2018d). It is also well known that the axial field strength and CME speed are correlated (Gonzalez et al., 1998; Gopalswamy et al., 2015b).

At lower frequencies $(<100 \mathrm{MHz})$, the radio emission lasted for about $30 \mathrm{~min}$ and contains several components: type III, type II, and a flare continuum. The flare continuum is weaker than the type II and type III bursts. The flare continuum is thought to extend to lower frequencies as DH type IV continuum (Gopalswamy et al., 2016a). Three episodes $(1,2,3)$ of type II bursts are observed, and whose fundamental (F1, F2, F3) and harmonic $(\mathrm{H} 1, \mathrm{H} 2, \mathrm{H} 3)$ components are marked in Figure 8 . The starting times and frequencies of the fundamental components (F1, F2, F3) are shown in Table 1. 


\subsubsection{NDA Type II Bursts from Shock Flanks}

The onset of episode 1 at 11:00:34 roughly indicates the shock formation time. This is consistent with the EUV wave observed around 11:00:22 UT (Grechnev et al., 2018). From the height-time history of the shock in Figure 6, we infer the shock height and speed to be $1.82 \mathrm{Rs}$ and $1269 \mathrm{~km} \mathrm{~s}^{-1}$ at the time of episode 1 . The burst had a clear fundamental and harmonic structure and well-defined starting frequency $(45.1 \mathrm{MHz})$ of the fundamental. The sharp cutoff of the radio emission at $180 \mathrm{MHz}$ suggests that plasma levels above $90 \mathrm{MHz}$ are occulted. Therefore, the type II emission at $45 \mathrm{MHz}$ is unocculted. According to Newkirk's density model above active regions, the density is given by $n\left(\mathrm{~cm}^{-3}\right)=n_{0} 10^{4.32 / r}$, where $r$ is the heliocentric distance in units of Rs, and $n_{0}=2 \times 10^{4}$. This distribution is equivalent to a power-law distribution $n \sim r^{-6.59}$, indicating a steep drop in density as a function of distance in the corona. Gopalswamy et al. (2013b) found a similar steep decline, $r^{-7.56}$, based on EUV observations in the early part of solar cycle 24. According to these models, the plasma level corresponding to the type II starting frequency of $45.1 \mathrm{MHz}$ should be at a heliocentric distance of 1.75 and $1.66 \mathrm{Rs}$, respectively. These values are close to the measured shock height of 1.82 Rs using the model fit, suggesting that the type II source is likely to be within $15^{\circ}-24^{\circ}$ from the shock nose. The drift rate $(d f / d t)$ of the type II burst was $0.13 \mathrm{MHz} \mathrm{s}^{-1}$, which is typical of metric type II bursts (Mann et al., 1996; Gopalswamy et al., 2009b). The shock speed V can be derived from the drift rate using the relation,

$$
V=2 L(1 / f)(d f / d t)
$$

where $L=\left|n(d n / d r)^{-1}\right|$ is the density $(n)$ scale height and $\mathrm{f}$ is the emission frequency (equal to the local plasma frequency). For a power-law distribution of the form

$$
n=n_{0} r^{-\alpha}, \quad \text { with } L=r / \alpha
$$

so we get $V=1076 \mathrm{~km} \mathrm{~s}^{-1}$. Taking into account of the possibility that the source may be slightly away from the nose, the speed becomes $1114 \mathrm{~km} \mathrm{~s}^{-1}$, close to the local shock speed $\left(1269 \mathrm{~km} \mathrm{~s}^{-1}\right)$. If the emission comes from the nose, the drift rate relation can be used with $V=1269 \mathrm{~km} \mathrm{~s}^{-1}$ to get $L=0.33 \mathrm{Rs}$. Since $r=1.82 \mathrm{Rs}$, we get $\alpha=5.59$, which is also reasonable $\left(n_{0}=7.14 \times 10^{8} \mathrm{~cm}^{-3}\right)$.

The type II episodes F2 and F3 occur when the shock nose is in the height range 3.5 to $4 \mathrm{Rs}$, moving with a very high speed $\left(>2000 \mathrm{~km} \mathrm{~s}^{-1}\right)$. The plasma frequencies at these heights are in the range 7.3 to $5 \mathrm{MHz}$. While $\mathrm{DH}$ type II emission is present at these frequencies, the type II emission at 32.9 and $25.6 \mathrm{MHz}$ cannot come from the shock nose. According to the density distribution noted above $\left(n \sim r^{-5.59}\right), 32.9$ and $25.6 \mathrm{MHz}$ plasma levels occur at distances 2.04 and 2.23 Rs, respectively. This means, these type II bursts come from distant flanks of the shock, about $56^{\circ}$ away from the shock nose. The flank speed is expected to be $>1000 \mathrm{~km} \mathrm{~s}^{-1}$, which is high enough to support a shock. Note that different type II episodes might originate from different parts of the three-dimensional shock surface that cuts the appropriate plasma level. Episode 2 had an initial positive slope, which is likely due to the flank crossing a high-density structure such as a streamer. The Radio Solar Telescope Network (RSTN) reported a type II burst in the range 53-25 MHz during the interval 11:13 to 11:24 UT, which overlaps with episodes 2 and 3. Episode 1 was not reported by RSTN. The complex nature of the metric type II episode is typical of energetic eruptions in which many sections of the shock front is likely to produce type II bursts (see e.g., the 2017 September 10 eruption reported in Gopalswamy et al., 2018c). 


\subsubsection{Heliocentric Distance of the Shock at SGRE End}

The IP type II burst can be used to determine the heliocentric distance of the shock until which it was accelerating $>300 \mathrm{MeV}$ protons in sufficient numbers to produce SGRE. For this we compute the drift rate of the type II burst over a stretch of the burst before the first break in the dynamic spectrum around 16:15 UT (see Figure 7). Between 13:54 and 16:12 UT, the fundamental component of the burst drifts from $0.43 \mathrm{MHz}$ to $0.31 \mathrm{MHz}$, yielding a drift rate of $1.35 \times 10^{-5} \mathrm{MHz} \mathrm{s}^{-1}$. From the height-time plot of the shock in Figure 6 , the shock speed at 12:10 UT was $\sim 2300 \mathrm{~km} \mathrm{~s}^{-1}$ and was slowly decreasing at the rate of $0.027 \mathrm{~km} \mathrm{~s}^{-2}$. This average deceleration would bring down the shock speed to $\sim 1900 \mathrm{~km} \mathrm{~s}^{-1}$ at 16:12 UT.

We can obtain the heliocentric distance over which the shock sends particles to the Sun to produce $\gamma$-rays. We select 16:12 UT as the reference time, which is close to the break in the type II burst noted above. At this time, $V=1900 \mathrm{~km} \mathrm{~s}^{-1}, L=r / 2(\alpha=2$ in the IP medium), $f=0.31 \mathrm{MHz}$, and $d f / d t=1.35 \times 10^{-5} \mathrm{MHz} \mathrm{s}^{-1}$. Substituting these quantities in Equation 1, we get $r=62.7$ Rs at 16:12 UT. Another way is to uses the shock height measured from the GCS fit and extrapolate it to 16:12 UT using the density fall off (Equation 2). From the height-time plot in Figure 6 we see that at 12:10 UT, the shock was at a height of $15 \mathrm{Rs}$ and the plasma frequency is $\sim 1.2 \mathrm{MHz}$, which corresponds to a plasma density of $1.78 \times 10^{4} \mathrm{~cm}^{-3}$. The local plasma density at $16: 12$ UT is given by the plasma frequency $0.31 \mathrm{MHz}$ as $1186 \mathrm{~cm}^{-3}$. If the density varies as $r^{-2}$, we can get the shock distance from the plasma densities at 12:10 UT and 16:12 UT as 58.1 Rs, which is similar to the one derived from the drift rate and differs only by $\sim 7.3 \%$. This result is consistent with the shock heliocentric distance of $\sim 90$ Rs at the end of the 2015 June 21 SGRE reported in Gopalswamy et al. (2018b).

\subsection{SEP Association}

Figure 9 shows the proton intensity observed by GOES as a function of time over several days. The peak SEP flux in the $>10 \mathrm{MeV}$ energy channel is $<10 \mathrm{pfu}$, making it a minor event at Earth. The onset of the SEP event is delayed by $\sim 9$ hrs because of the poor connectivity to Earth (the well-connected field lines are about $170^{\circ}$ away from the source region). The SEP intensity is roughly the same in all three GOES energy channels (>10 MeV, $>50 \mathrm{MeV}$, and $>100 \mathrm{MeV}$ ) until about $16 \mathrm{UT}$ on September 2, suggesting a hard spectrum. The intensity decayed extremely slowly in all three channels: until September 7 in the $>100 \mathrm{MeV}$ channel and until September 10 in the other two channels. A similar longenduring GOES particle intensity was observed during the backside extreme event of 2012 July 23 (Gopalswamy et al., 2016b). The height-time plots of all the CMEs are also shown in Figure 9 (middle). It is clear that the only full halo CME is the CME in hand. None of the CMEs had any effect on the intensity curves, except the one on September 5. The GOES plot at the bottom indicates that the flare is occulted in the Sep14 event.

Figure 10a shows the revised SGRE source location along with the EUV wave front on the solar disk (pointed by arrows). Note that the EUV front corresponds to the ground track of the EUV wave dome represented by the spheroid in Figure 5. Thus, the SGRE source location corresponds to the field lines located between the active region site and the shock flank. Since STB was well-connected to the source, an intense SEP event was detected by that spacecraft. The proton intensity in Figure $10 \mathrm{~b}$ in the $62-100 \mathrm{MeV}$ energy channel sharply rises with the eruption and slowly decays over a few days, consistent with the GOES intensity profile. The SGRE event lasted roughly until the declining phase of the first peak 

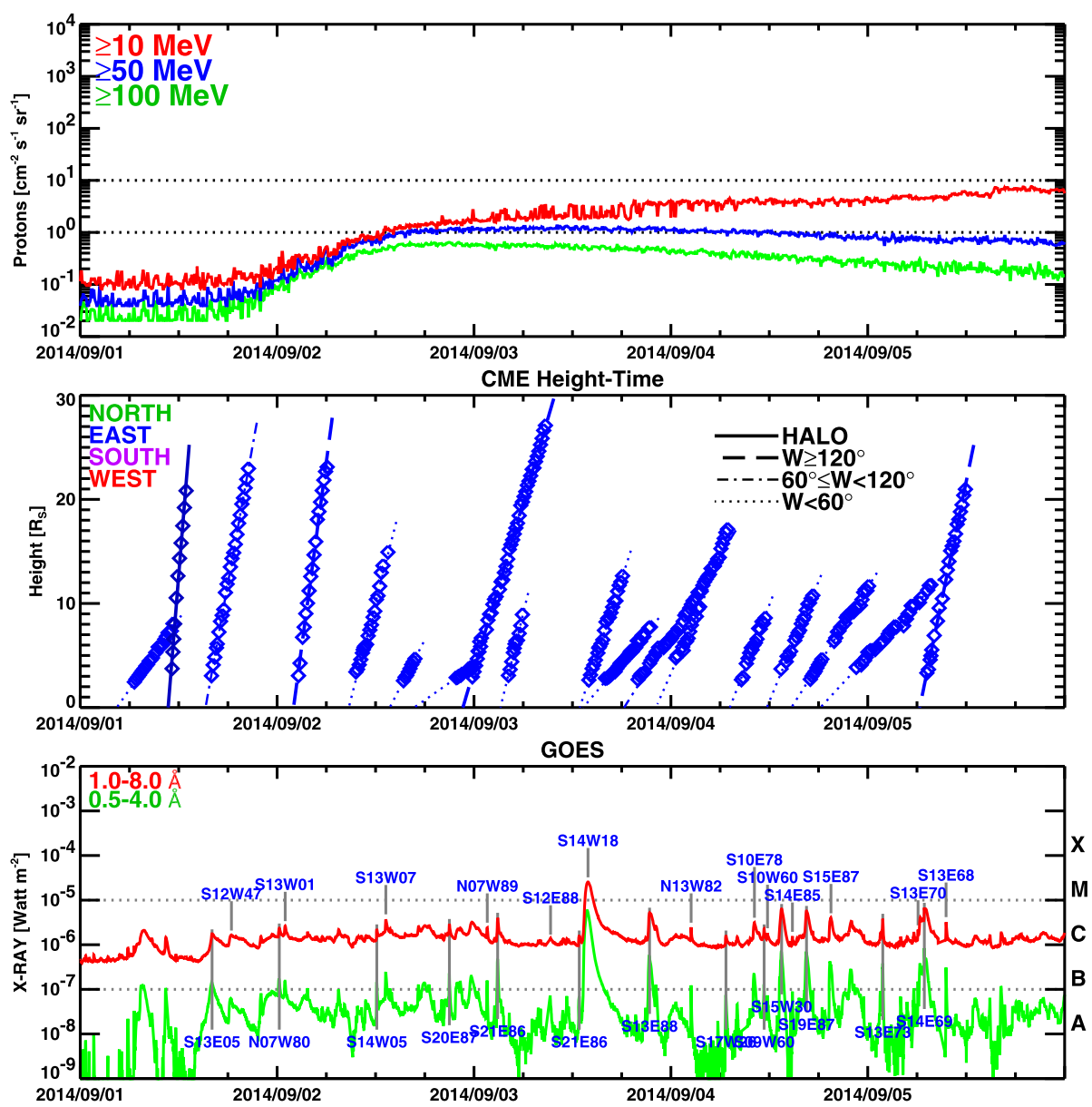

Figure 9 SEP, CME, and flare activities observed over the interval 2014 September 1-5 from the Sun-Earth line. (top) GOES protons flux in three integral energy channels, $>10 \mathrm{MeV}$ (red), >50 MeV (blue), and $>100 \mathrm{MeV}$ (green). (middle) CME height-time plots for CMEs heading in the east direction. (bottom) GOES soft X-ray intensity as a function of time. In the middle panel, only eastern CMEs are included. There was no CME from the western hemisphere with SEP association. Only the Sep14 CME was a halo CME.

in the $62-100 \mathrm{MeV}$ intensity profile observed at STB. The particle event was also detected at STA, which was located at W166, but only briefly due to large data gaps. The intensity was consistent with the STB curve, although a bit lower during the brief period (centered at 8 UT on September 2).

\subsubsection{Fluence Spectrum}

We now confirm that the Sep14 SEP event had a hard spectrum, using STEREO data (10$100 \mathrm{MeV}$ ), consistent with the spectrum obtained from the Payload for Matter-Antimatter Exploration and Light Nuclei Astrophysics (PAMELA) data at energies $>80 \mathrm{MeV}$ (Bruno et al., 2018) PAMELA detector recorded SEPs up to $700 \mathrm{MeV}$. De Nolfo et al. (2019a) determined that the Sep14 event had event-integrated intensity at $>500 \mathrm{MeV}$ as $1.5 \times$ 


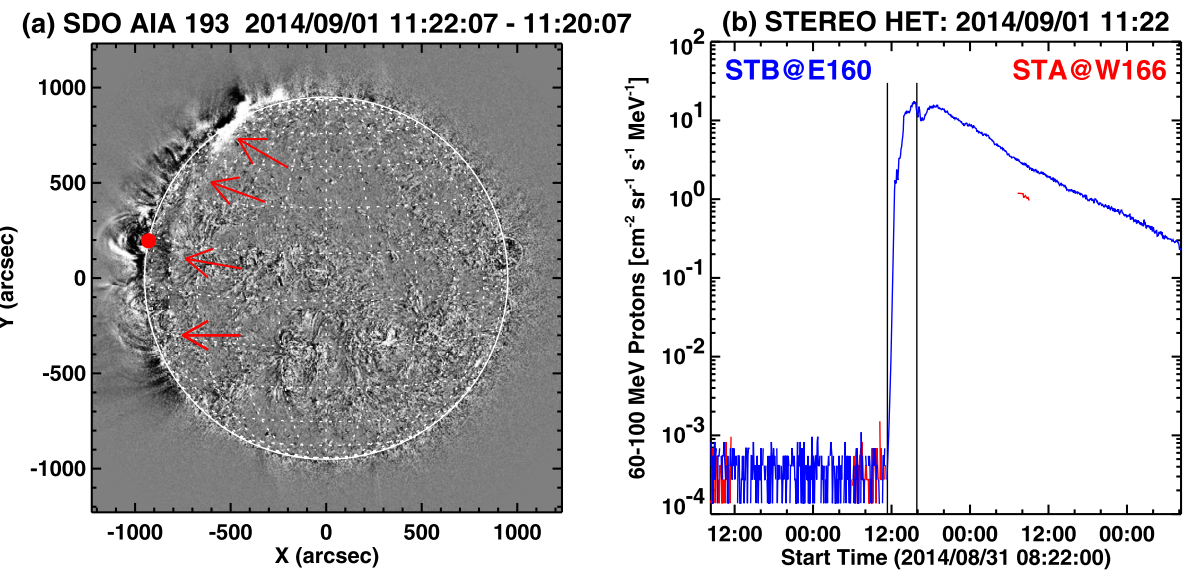

Figure 10 (a) EUV shock on the solar disk (along E60 marked by arrows) propagating away from the backside source as seen in a EUV difference image at 11:22:07 UT. The red dot at the limb is the location of SGRE at 11:15 UT revised from Ackermann et al. (2017). (b) Plot of proton flux in the 62-100 MeV energy channel of STB/HET (blue). STA had data gap except a brief moment (red). The first vertical line marks the time of the SDO/AIA image on left (11:22 UT). The second vertical line marks the end of SGRE (15:52 UT). The STA and STB were at W166 and E160, respectively from Earth view.
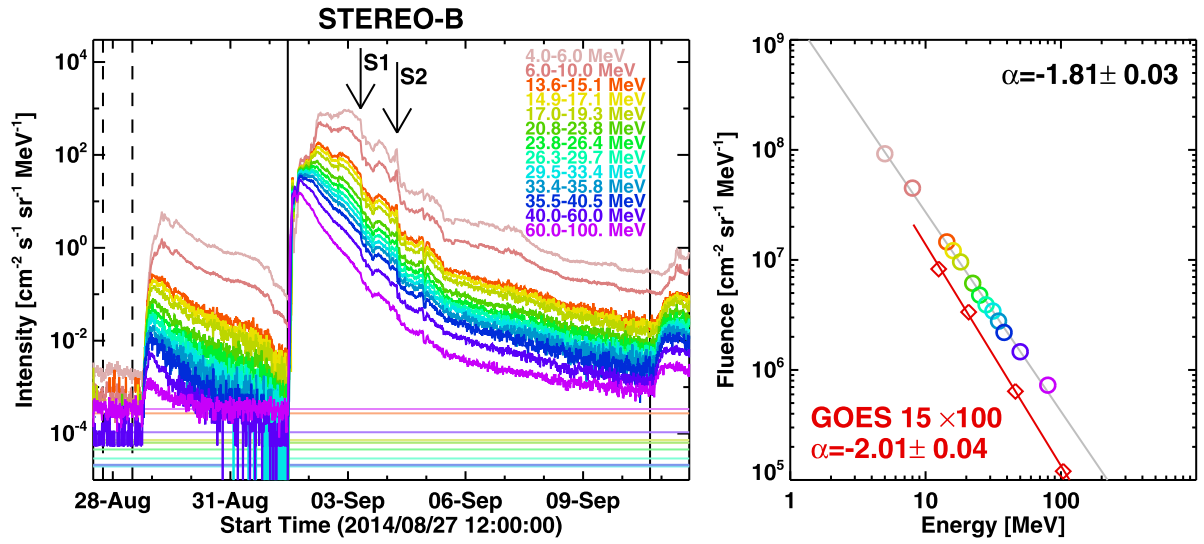

Figure 11 (left) Proton intensities at various energy channels from STB. The 4-6 MeV and 6-10 MeV data are from STB/LET and the remaining are from STB/HET. The fluences were determined over the interval between the vertical dark lines. The background intensity of protons was obtained as an average over the interval marked by the vertical dashed lines (from 18:00 UT on 2014 August 27 to 12:00 UT on 2014 August 28) to avoid preceding SEP event from a different source region. S1 (September 3 at 07:45 UT) and S2 (September 4 at 06:07 UT) are the shocks detected in situ at STB; S1 is associated with the CME in question, indicating a transit time of $\sim 45 \mathrm{hr}$. (right) The fluence spectrum from STB. The spectrum is fit to a power law (gray line) with an index of $1.81 \pm 0.03$. The red line is the spectrum obtained from GOES data. The GOES fluences are multiplied by a factor 100 and fit to a power law with an index of $2.01 \pm 0.04$.

$10^{3} \mathrm{~cm}^{-2} \mathrm{sr}^{-1}$. Figure 11a shows the SEP intensity at various STEREO energy channels and the fluence values in each of these channels. The event in question was preceded and followed by other SEP events, so the fluence computed is approximate, especially because it is difficult to decide the background level. However, choosing background levels at dif- 
ferent intervals roughly resulted in the same fluence spectrum. The derived fluence spectrum is extremely hard, at the theoretical limit of 2 . Figure $11 \mathrm{~b}$ shows the spectrum with a fit to the 10-100 MeV data points from STEREO LET and HET. The spectral index is -1.81 , close to the theoretical value for diffusive shock acceleration (see, e.g., Vainio, 2009; Wolff and Tautz, 2015). The GOES HEPAD data also yields a hard spectrum with a spectral index of $\sim-2.01$, in good agreement with the STEREO data. The spectrum is thus one of the hardest, similar to well-connected GLE events. Cohen and Mewaldt (2018) found this event to be an extreme event with one of the hardest fluence spectrum. In our previous work (Gopalswamy et al., 2018a), we noted that the lack of $>300 \mathrm{MeV}$ particles at Earth in the 2011 March 07 SGRE event is due to poor latitudinal connectivity (the shock nose is too far above the ecliptic) because of the source location (N31) and an unfavorable solar B0 angle $\left(-7^{\circ} .25\right)$ (see Gopalswamy et al., 2013a; Gopalswamy and Mäkelä, 2014). The Sep14 event had the opposite situation: The source latitude was N14, while the B0 angle is $+7^{\circ} .25$, so the effective source location with respect to the ecliptic was N07, well within the average nose distance of GLE events $\left(\sim \pm 13^{\circ}\right)$. Accordingly, STB observed a large SEP event and the computed $>100 \mathrm{MeV}$ proton intensity remained high during the SGRE event. Even though the highest HET energy channel is $62-100 \mathrm{MeV}$, we infer that there were particles of significantly higher energy, possibly up to $\mathrm{GeV}$ energies.

\subsubsection{Event Size at $>10 \mathrm{MeV}$ Energies}

The high intensity of the event at STB is evident from the "snow storm" visible in Figures 3 and 4. In fact, STB/COR1 images had the snow storm at least until the end of September 3 (see https://cdaw.gsfc.nasa.gov/stereo/daily_movies/2014/09/03/). We computed the $>10 \mathrm{MeV}$ intensity at STB from the spectrum in the 10-100 MeV range every $60 \mathrm{~min}$ under the assumption that there are no particles with energies $>1 \mathrm{GeV}$. We average the data over $60 \mathrm{~min}$ because the spectrum was highly variable over shorter intervals due to velocity dispersion early in the event. We use the following procedure to get the $>10 \mathrm{MeV}$ flux. STEREO/HET records data in the energy range 13.6 to $100 \mathrm{MeV}$ in 11 energy channels. The observations need to be extended to $1000 \mathrm{MeV}$ on the higher energy side and to $10 \mathrm{MeV}$ on the lower energy side. First, we estimated the flux in the HET energy range 13.6 to $100 \mathrm{MeV}$ by fitting a power law to the observations. Second, we obtain the 100$1000 \mathrm{MeV}$ flux assuming the power-law index to be 1.81 (same as the power-law index of the fluence spectrum in Figure 11b) that matches the flux in the 60-100 MeV channel until about 04:30 UT on September 2; beyond that, the actual spectral indices obtained from all HET channels are used. Third, we obtain the flux in the 10 to $13.6 \mathrm{MeV}$ in two ways: (i) assuming the power law fitted to the HET data is valid down to $10 \mathrm{MeV}$, and (ii) interpolating between the highest energy channel in LET (6-10 MeV) and the lowest energy channel in HET (13.6-15.1 MeV). The background flux is subtracted in all cases. By adding the fluxes in the three steps above gives the $>10 \mathrm{MeV}$ flux. Figure 12 (left) shows the $>10 \mathrm{MeV}$ flux obtained. The flux obtained from HET data alone gives a $>10 \mathrm{MeV}$ peak flux of $4170 \mathrm{pfu}$. Combining the LET and HET data gives a $>10 \mathrm{MeV}$ peak flux of $\sim 3564$ pfu. The peak fluxes differ only by $\sim 17 \%$, suggesting that the Sep14 event is truly an intense event. The derived $>10 \mathrm{MeV}$ flux at STB is thus larger than that at GOES by more than two orders of magnitude.

Figure 12 (right) shows the time evolution of the $>100 \mathrm{MeV}$ STB proton flux computed by extrapolating the $10-100 \mathrm{MeV}$ spectrum to higher energies assuming that the power-law index of the fluence spectrum is also applicable to 1-hour intervals and assuming that the number of particles with energy $>1 \mathrm{GeV}$ is negligible. The $>100 \mathrm{MeV}$ proton flux serves as 

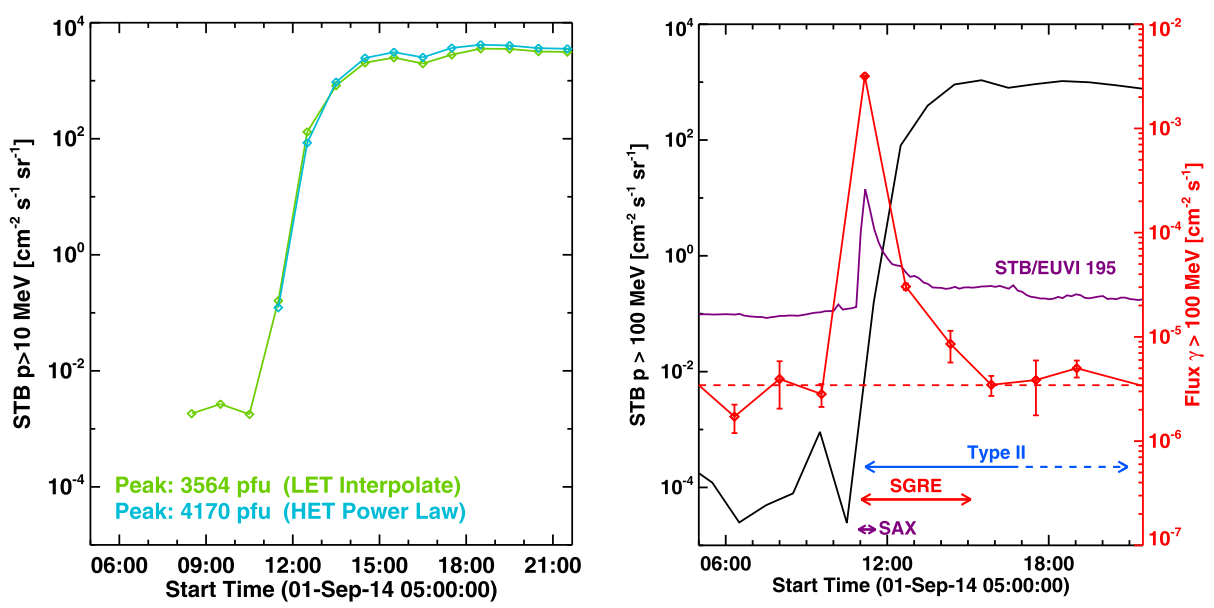

Figure 12 (left) Time profiles of the $>10 \mathrm{MeV}$ proton flux obtained from STB data with two different ways of estimating the flux between 10 and $13.6 \mathrm{MeV}$ : (i) Using HET data alone by fitting a power law to the HET data and assuming that the power law is valid down to $10 \mathrm{MeV}$ (red) and (ii) interpolating between the last (6-10 MeV) LET data point and first (13.6-15.1 MeV) HET data point (blue). The peak fluxes obtained by the two methods are shown on the plot. (right) Time profiles of the $>100 \mathrm{MeV}$ proton flux from STB (black curve), $>100 \mathrm{MeV}$ SGRE flux (red curve) and the flare represented by the STB/EUVI intensity (purple curve in arbitrary units). In obtaining the $>100 \mathrm{MeV}$ proton flux, we started from the 60-min averaged flux in the 60-100 MeV HET channel and used the power-law index of the fluence spectrum (1.81) to extend up to $1 \mathrm{GeV}$. The horizontal dashed red line marks the $\gamma$-ray background. The duration of the type II burst from SWAVES dynamic spectrum and the SGRE duration are marked by the blue and red double arrows, respectively. The purple double arrow marks the duration (10:55 UT to 11:34 UT) of the soft X-ray flare derived from the MESSENGER SAX instrument (from Share et al., 2018).

a proxy to the $>300 \mathrm{MeV}$ proton flux required for SGRE. Also shown is the $>100 \mathrm{MeV} \gamma-$ ray flux from Fermi/LAT. The $>100 \mathrm{MeV}$ proton flux increased by four orders magnitude around the onset of SGRE and remained high throughout the event. The SGRE duration is longer than all impulsive-phase activities and the end of soft X-ray flare emission and closer to the type II burst duration. The intensity of the flare in EUVI (STB), shown as a proxy to the soft X-ray flare emission coincides with the SGRE peak (within $\sim 5$ min time resolution of the EUVI observations). Share et al. (2018) used the soft X-ray count rates from the MESSENGER SAX instrument to identify the equivalent GOES flare duration between 10:56 UT and 11:34 UT, with a peak at 11:10 UT coincident with the STB/EUVI light curve.

\subsection{Duration Comparisons}

Gopalswamy et al. (2018a) reported a linear relationship between the durations of SGRE events $\left(T_{\mathrm{SGRE}}\right)$ and type II bursts $\left(T_{\mathrm{II}}\right)$ for a set of 13 SGRE events. They had also shown the 1991 June 11 SGRE event from Kanbach et al. (1993), but not included in the correlation. In Figure 13, we show the $T_{\mathrm{SGRE}}-T_{\mathrm{II}}$ plot that includes the 1991 June 11 event. The best fit to the scatter plot gives the following relation (all durations are in units of hours):

$$
T_{\mathrm{SGRE}}=(1.0 \pm 0.2) T_{\mathrm{II}}+(0.1 \pm 2.1)
$$

This relation is not too different from the one in Gopalswamy et al. (2018a), confirming the linear relationship. We have over plotted the Sep14 data point in Figure 13 with $T_{\mathrm{SGRE}}=$ 

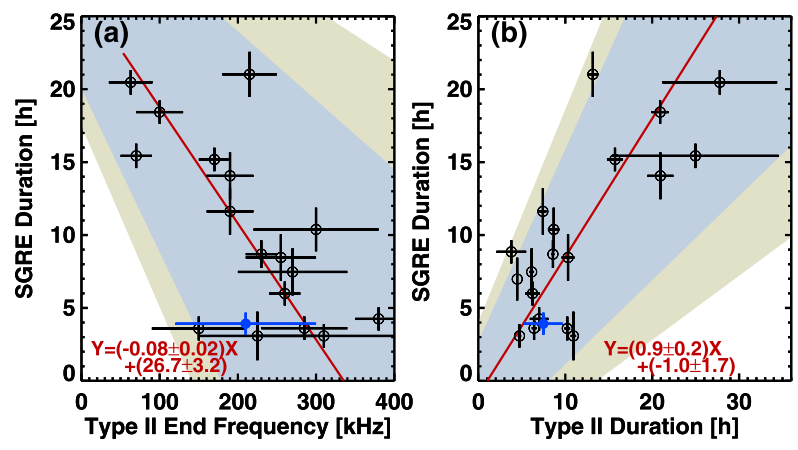

Figure 13 The SGRE duration, type-II ending frequency, and type II duration of the Sep14 event (blue data points) compared with 14 SGRE events reported in Gopalswamy et al. (2018a). The red lines in (a) and (b) are the best-fit lines for the 14 events. The blue and yellow shaded areas denote the $95 \%$ and $99 \%$ confidence intervals of the fit. The blue data points were not included in the fit but are consistent with the linear relationships in (a) and (b). Note that the Sep14 SGRE duration is expected to be a lower limit, so the agreement is likely better when the actual duration of SGRE is considered (the blue data point would move up toward the regression lines in (a) and (b)).

$3.92 \pm 0.76 \mathrm{hr}$ and $T_{\mathrm{II}}=7.5 \pm 2.25 \mathrm{hr}$. The ending frequency of the type II burst is $210 \pm$ $90 \mathrm{kHz}$. The Sep14 event agrees with the two best-fit lines in Figure 13. Note that we have used a generous error bar for the type II burst duration, taking the break at 16:18 UT and the end at 20:56 UT as possible end times. It must be noted that the SGRE duration given in Figure 1 is likely to be an underestimate because we are not observing the entire flux of the $\gamma$-rays. There must be protons precipitating into other parts of the chromosphere that have magnetic connectivity to the shock nose. This limits the flux of gamma rays, and hence the last SGRE data point. Ackermann et al. (2017) compared the Sep14 event with disk events of similar flare size. They found that the total $\gamma$-ray energy is greater for on-disk flares probably due to the fact they are observed over longer timescales. Any increase in SGRE duration would move the data points in both plots in Figure 13 closer to the best-fit lines. Thus, we can conclude that the Sep14 SGRE event is consistent with other SGRE events with a similar duration. Furthermore, Gopalswamy et al. (2019) extended the scatter plot to include SGRE events with duration exceeding $3 \mathrm{~h}$. The resulting best-fit line is given by $T_{\mathrm{SGRE}}=(0.9 \pm 0.2) T_{\mathrm{II}}+(-0.8 \pm 1.9)$, which is not too different from the one given by Equation 3.

\section{Discussion}

The detailed investigation presented in this paper focused on how the SGRE event is related to the eruption geometry, 3D kinematics of the CME and shock, SEP event, and various types of radio bursts. The type II burst extending to very low frequencies is a signature of a strong shock, which is confirmed by the ultra-fast CME (speed $>2000 \mathrm{~km} \mathrm{~s}^{-1}$ ). The SEP spectrum is very hard-typical of GLE events. The hard spectrum is confirmed using GOES and STB data and is consistent with the observation of high-energy particles by PAMELA (Bruno et al., 2018). Gopalswamy et al. (2016b) reported the fluence spectral indices of 86 western SEP events from Solar Cycles 23 and 24. The spectral index ranged from 2.01 to 6.12. The spectral index of only four events is similar to that of the Sep14 event (index < 2.1): 1997 November 06 (2.07), 1998 May 02 (2.01), 2006 December 13 (2.07), 
and 2012 July 08 (2.01). The first three are GLE events and the last one is a large SEP event. A reanalysis of the last event showed that the spectral index is slightly larger, $\sim 2.6$. Thus, we are confident that the 2014 September 01 event is similar to GLE events, indicating that $>300 \mathrm{MeV}$ particles required by the SGRE event are definitely present.

\subsection{The Magnetic Structure Associated with SGRE}

In this section, we develop a schematic model based on these observations and their synthesis into a coherent picture. In particular, we focus on how the temporally and spatially extended nature of the SGRE event follow naturally from the observations, especially when combined with the possible magnetic structures involved and how they are accessed by energetic particles accelerated at the flare site and the shock front. We attribute the temporally extended nature to the continued acceleration of high-energy particles by the CME-driven shock. The large spatial extent is determined by the footprint of the shock sheath that contains the open field lines surrounding the flux rope and threading through the shock nose. The SGRE source extension is naturally much larger than the post-eruption arcade to which the impulsive-phase gamma rays are confined.

One of the main characteristics of the SGRE events is that the $\gamma$-ray emission is temporally distinct from the impulsive-phase emission (Share et al., 2018). The 2014 September 01 event showed that the SGRE emission is likely to be spatially distinct from the impulsivephase emission. While the impulsive-phase emission is confined to the post-eruption arcade, the extended phase $\gamma$-rays are associated with a structure much larger than the PEA.

A typical solar eruption involves two closed magnetic structures: the PEA and the flux rope, both of which are thought to be formed during the reconnection process. The PEA remains anchored to the solar surface, while the flux rope is ejected. The flux rope is also anchored to the solar surface, but the separation between the flux rope feet is larger than the extent of the PEA (see Figure 14 and Webb et al., 2000; Gopalswamy, 2009). The large extent of the flux rope is readily inferred from the fact that all SGRE events with duration $>3 \mathrm{hr}$ are associated with halo CMEs (Gopalswamy et al., 2018a, 2019) and halo CMEs are inferred to be fast and wide from quadrature observations (Gopalswamy et al., 2013c).

Particles accelerated during the reconnection process have access to both these structures. Electrons entering the PEA produce various microwave bursts and stationary type IV bursts (also known as flare continuum) in radio and hard X-ray bursts, while protons produce the impulsive-phase $\gamma$-ray bursts. The spatial extent of the impulsive-phase emissions is defined by the size of the PEA. Electrons trapped in the flux rope produce moving type IV bursts. As the flux rope expands and moves away from the Sun, the electrons lose energy and the moving type IV burst decays. When the flux rope is super-Alfvenic, it drives a fastmode MHD shock, which stands off from the flux rope at a distance determined by the radius of curvature of the flux rope and the shock Mach number (see, e.g., Gopalswamy and Yashiro, 2011). Open magnetic field lines surrounding the flux rope thread through the shock and form the third magnetic structure of interest. The schematic in Figure 14 shows these magnetic structures. The PEA with dimming regions has been observed during the 2015 June 21 SGRE event and a flux rope was reconstructed using white-light observations (Gopalswamy et al., 2018b, their Figures 5 and 13).

The shock is another source of energetic particles that have access to the open field lines threading through the shock and hence travel both toward and away from the Sun. The shock-accelerated particles thus occupy a volume much larger than that of the PEA and flux rope and surround the flux rope. Electrons escaping the shock front produce type II radio bursts at the local plasma frequency and harmonics. The bursts can start typically at a 


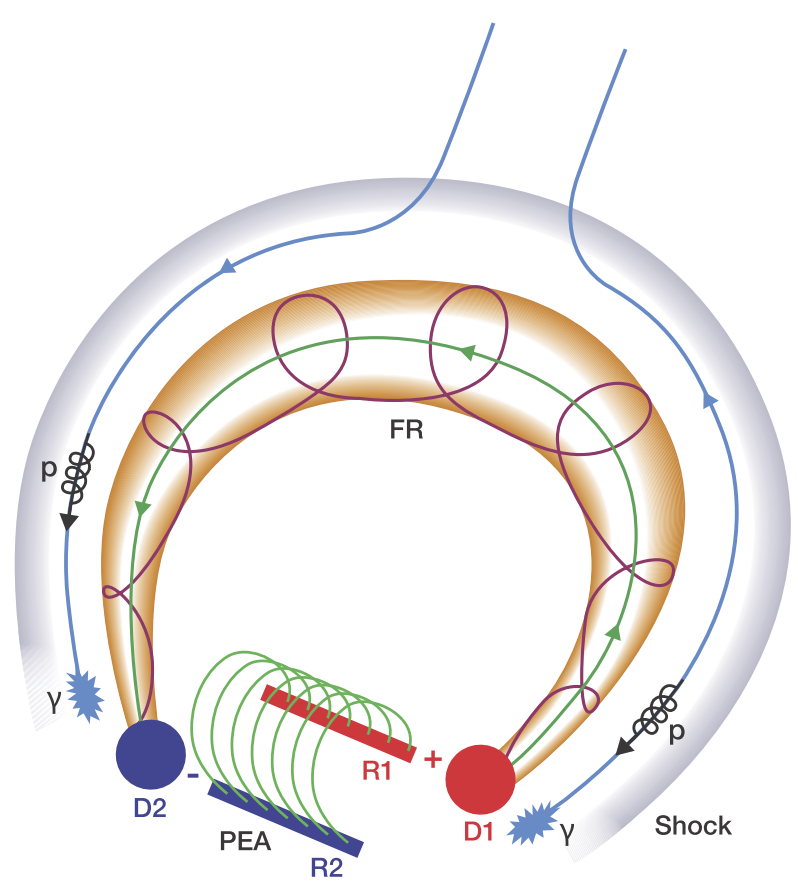

Figure 14 A schematic showing the magnetic structures involved in a typical eruption. The post-eruption arcade (PEA) with its feet rooted on the flare ribbons R1 and R2. The flux rope (FR) is a much larger structure rooted in the dimming regions D1 and D2. The flux rope is surrounded by a shock dome. Note that all these structures are directly inferred from observations during the 2014 September 01 event. Open magnetic field lines (represented by the blue lines) in the sheath region that thread through the shock nose are rooted in the region beyond the dimming regions. Protons $(>300 \mathrm{MeV}$, black coils) accelerated at the shock front travel along these open field lines precipitate to the solar chromosphere (indicated by the patches at the bottom of the blue lines) and produce $\gamma$-rays; the separation between the patches represents the largest spatial extent of the SGRE source.

frequency of $\sim 150 \mathrm{MHz}$ and extend to lower frequencies, ending at different frequencies, depending on the strength of the shock. Type II bursts extending to very low frequencies $(\sim 20 \mathrm{kHz})$ close to the local plasma frequency at the observing spacecraft indicate the strongest of shocks that also result in large SEP events (Gopalswamy, 2006). Type II bursts are produced typically by $\sim 10 \mathrm{keV}$ electrons via the plasma emission mechanism, while higher-energy electrons are observed in-situ as electron events. Higher-energy electrons can also propagate toward the Sun and may contribute to bremsstrahlung continuum. Protons accelerated by the shock are detected in situ as SEP events, while those propagating toward the Sun produce $\gamma$-rays (SGRE) if the proton energy exceeds $300 \mathrm{MeV}$.

Figure 14 sketches the scenario of $>300 \mathrm{MeV}$ protons streaming toward the Sun and precipitation to produce SGRE. The protons are accelerated in the vicinity of the IP shock driven by the CME flux rope. It is implied that the legs of the CME flux rope are rooted on the Sun at the core-dimming regions on either side of the neutral line, but outside the post-eruption arcade. Open field lines threading the shock-nose intersect the solar surface just outside the flux rope. SGRE events require $>300 \mathrm{MeV}$ protons, which are generally accelerated closer to the nose, where the shock is the strongest. In this sense, the scenario depicted in Figure 14 can be thought of as a variant of the cartoon model in Cliver, Kahler, and Vestrand (1993), where protons from the shock flanks precipitate to the photosphere to 
produce the $\gamma$-ray line emission, which requires lower proton energies. The extent of the shock nose and its geometry is expected contribute significantly to the $\gamma$-ray variability in eruptive events. In particular, the orientation of the flux rope is likely to play a significant role in the varying shock-nose extent. Future $\gamma$-ray missions with a better spatial resolution should be able to discern the spatial distribution of $\gamma$-rays around the eruption region.

Recently Hudson (2018) criticized the CME-shock solution by suggesting that a strong mirror force prevents protons from propagating sufficiently close to the Sun. The mirroring is a common problem to particles precipitating along any magnetic structure: PEA, flux rope, or the open structures threading the shock nose. It must be noted that in the case of PEA, particles precipitate from high magnetic field regions to higher magnetic field regions. On the other hand, in the case of particles precipitating along open field lines, the precipitation occurs from low-field region ahead of the shock nose to the quiet-Sun field regions beyond the flux rope legs. Hudson (2018) suggested a "lasso" scenario, where accelerated protons are captured by closed magnetic fields that form a noose extending to heights of several solar radii. As the loop retracts in the aftermath of the CME, high-energy protons are transported by advection into denser solar atmosphere. In a typical eruption scenario, the flux rope structure is rapidly moving and expanding, while the PEA evolves slowly. It is not clear how the magnetic lasso is created and where it is placed with respect to the PEA, flux rope, and shock.

While the shock-sheath region naturally extends to the front-side, allowing protons to precipitate and produce pion-decay $\gamma$-rays, Grechnev et al. (2018) invoke a static magnetic field structure emanating from the eruption site and ending on the frontside of the Sun. They show this using potential field extrapolation using a pre-event synoptic magnetic chart. Photospheric magnetic fields seldom show changes due to eruption. Nonpotential fields possess the energy for the eruption, so potential field extrapolation cannot explain the eruption-associated disturbances in a violent event such as the Sep14 event. EUV images show another active region between the solar limb and the region of interest, so it is not clear if the structure emanated from the flaring region. Such a structure also does not fit in the clear PEA-flux rope structure observed and depicted in Figures 3-5. Therefore, it is not clear if such large structures may explain particle acceleration and trapping within such large coronal structures not causally connected to the CME shock trap and accelerate particles to produce the observed SGRE (De Nolfo et al., 2019a; De Nolfo et al., 2019b).

The hard X-ray data presented by Grechnev et al. (2018) has a weak emission in the energy range 161-195 KeV, which may be from the same population of electrons that produced the gyrosynchrotron emission peaking at $\sim 1000 \mathrm{MHz}$. Using a parametric fit to the radio flux density spectrum and analysis of X-ray emissions detected by Fermi's Gammaray Burst Monitor (GBM) Carley et al. (2017) inferred a common origin of non-thermal electrons responsible for the radio and X-ray emissions. The duration of the weak coronal hard X-ray emission is similar to that of the gyrosynchrotron sources and that of the DH type III burst. These emissions suggest that the flare-accelerated electrons are injected into the flux rope producing the hard X-ray and radio emissions. Particles from the same source but injected into the post-eruption arcade produce impulsive-phase emissions and the flare continuum at radio wavelengths. Particles accelerated at the shock do not have access to these two magnetic structures (PEA and flux rope), but to the field lines surrounding the flux rope.

\subsection{Proton Numbers Derived from SEP Events and SGRE}

We noted earlier that the gamma-ray flux in the Sep14 event is expected to be a lower limit because only that part of the emission originating from the frontside of the Sun is recorded 
by Fermi/LAT. Gamma rays produced at other precipitation sites are occulted. Ackermann et al. (2017) suggested that the energy in the $>500 \mathrm{MeV}$ protons might be underestimated in events that occur at heliocentric angles $>75^{\circ}$. Given the large size of the CMEs involved in SGRE events (half angle expected in the range $45^{\circ}-50^{\circ}$ ), it is likely that gamma-ray flux in events originating within $30^{\circ}$ from the limb are likely to be underestimated. Share et al. (2018) estimated the number of $>500 \mathrm{MeV}$ protons $\left(N_{\gamma}\right)$ needed to produce SGRE as $1.99 \times 10^{30}$. De Nolfo et al. $(2019 \mathrm{a}, 2019 \mathrm{~b})$ reported the number of $>500 \mathrm{MeV}$ protons $\left(N_{\text {SEP }}\right)$ observed in space by PAMELA to be two orders of magnitude higher: $2.35 \times 10^{32}$. Since $N_{\gamma}$ is likely to be underestimated for a limb/backside events, the difference between $N_{\gamma}$ and $N_{\mathrm{SEP}}$ is expected to be much smaller for the Sep14 event. Using a sample of 14 events, De Nolfo et al. (2019a, 2019b) concluded that $N_{\gamma}$ and $N_{\text {SEP }}$ are uncorrelated and that the $\gamma$-ray emission is probably not due to protons diffusing back to the Sun from CMEdriven shocks. However, if one accounts for the lack of nose connectivity in high-latitude events and the underestimate of $N_{\gamma}$, the correlation improves. A detailed report on the relation between $N_{\gamma}$ and $N_{\text {SEP }}$ taking into account of the connectivity issues in SEP events and the underestimate of $N_{\gamma}$ in limb events will be reported elsewhere.

Ackermann et al. (2017) reported that the total energy in $>100 \mathrm{MeV} \gamma$-rays is about two orders of magnitude smaller than that in $>500 \mathrm{MeV}$ particles. For the Sep14 event, the energy in the $>500 \mathrm{MeV}$ protons is 50 times larger than that in the $\gamma$-rays $\left(1.4 \times 10^{24} \mathrm{vs}\right.$. $7.0 \times 10^{25} \mathrm{erg}$ ). On the other hand, the energy in $>500 \mathrm{MeV}$ protons is seven orders of magnitude smaller than the CME kinetic energy. Using the observed CME mass of $\sim 1.6 \times 10^{16} \mathrm{~g}$ (https://cdaw.gsfc.nasa.gov/CME_list/UNIVERSAL/2014_09/univ2014_09.html) and the average speed in the coronagraph FOV of $\sim 2000 \mathrm{~km} \mathrm{~s}^{-1}$ (see Figure 5), we get a kinetic energy of $3.2 \times 10^{32} \mathrm{erg}$. Mewaldt et al. (2005) have shown that CME-shocks are as efficient as supernova shocks in accelerating particles in that $\sim 10 \%$ of CME kinetic energy goes into particle energy. It is clear that only a tiny fraction of the CME kinetic energy needs to go into the energy of $>500 \mathrm{MeV}$ protons to sustain the $\gamma$-rays. Furthermore, some numerical simulations and analytical work indicate significant excess of shock-injected protons as compared to the number of protons derived from SGRE observations (Kocharov et al., 2015; Afanasiev et al., 2018).

\section{Summary and Conclusions}

We performed a detailed investigation of the SOL2014-09-01 behind-the-limb eruption that resulted in the fourth largest SGRE event (in $>100 \mathrm{MeV}$ fluence) observed by Fermi/LAT. The fluence is likely a lower limit to the actual fluence because some $\gamma$-ray photons could not have reached Fermi/LAT. The eruption produced a twin dimming and a post-eruption arcade and a flux rope rooted in the dimming regions. The low-inclination flux rope implies an eruption with a large east-west extension. The flux rope had a three-dimensional speed exceeding $2000 \mathrm{~km} \mathrm{~s}^{-1}$ and was driving a fast shock. The shock-accelerated particles had a hard spectrum similar to previous GLE events. The shock was also the source of nonthermal electrons that produced a type II burst with emission at frequencies ranging from tens of $\mathrm{MHz}$ to tens of $\mathrm{kHz}$ suggesting an intense shock propagating in the interplanetary medium. The SGRE, CME, and radio burst properties of this event are consistent with all the disk events that had SGRE durations $\geq 3$ hrs. The main conclusions of the investigation are as follows.

(i) The SGRE and type II burst durations in the SOL2014-09-01 backside eruption are consistent with the linear relationship between the durations found in all $>3$-hr SGRE 
events. The SGRE duration and the ending frequency of the type II bursts are also consistent with an inverse relationship between the two quantities obtained before.

(ii) The magnetic structures in the eruption (post eruption arcade, flux rope, shock, and shock sheath) inferred from observations suggest a large east-west extent making it possible for the presence of the particle-precipitation region on the frontside solar disk in Earth view.

(iii) The largest extent of the SGRE source is inferred as the separation between the shock sheaths at the flanks of the flux rope. This means if limb is within the face-on angular half width of a behind the limb flux rope, one can still see $\gamma$-rays on the frontside.

(iv) The large extent of the SGRE source implies that part of it is hidden in the case of limb events.

(v) The CME/shock kinematics, the hard SEP spectrum, and the association with type II radio bursts over a wide range of wavelengths imply a very energetic CME with copious production of high-energy (up to $\mathrm{GeV}$ ) particles needed for the production of SGRE via neutral pion decay. This is consistent with the fact that some $>1 \mathrm{GeV} \gamma$-ray photons were observed that require multi-GeV protons.

(vi) The SGRE ended when the shock reached a heliocentric distance of $\sim 60$ Rs, consistent with previous studies.

(vii) The equivalent flux of $>10 \mathrm{MeV}$ SEPs computed from STEREO particle spectra, is $>3000 \mathrm{pfu}$, which makes it one of the largest SEP events in Solar Cycle 24.

Acknowledgements This work benefited from NASA's open data policy in using Fermi, Wind, SOHO, SDO, and STEREO data. We thank NOAA for making GOES X-ray and particle data available online. SOHO is a project of international collaboration between ESA and NASA. STEREO is a mission in NASA's Solar Terrestrial Probes program. CALLISTO data are made available online at e-callisto.org by the Institute for Data Science FHNW Brugg/Windisch, Switzerland (PI: C. Monstein). Orfées is part of the FEDOME project, partly funded by the French Ministry of Defense. We thank the Nançay Radio Observatory for making NDA data available online at http://www.obsnancay.fr. Work supported by NASA's Living with a Star program. HX was partially supported by NASA HGI grant NNX17AC47G. We thank the anonymous referee for helpful comments.

Disclosure of Potential Conflicts of Interest The authors declare that they have no conflicts of interest.

Publisher's Note Springer Nature remains neutral with regard to jurisdictional claims in published maps and institutional affiliations.

Open Access This article is licensed under a Creative Commons Attribution 4.0 International License, which permits use, sharing, adaptation, distribution and reproduction in any medium or format, as long as you give appropriate credit to the original author(s) and the source, provide a link to the Creative Commons licence, and indicate if changes were made. The images or other third party material in this article are included in the article's Creative Commons licence, unless indicated otherwise in a credit line to the material. If material is not included in the article's Creative Commons licence and your intended use is not permitted by statutory regulation or exceeds the permitted use, you will need to obtain permission directly from the copyright holder. To view a copy of this licence, visit http://creativecommons.org/licenses/by/4.0/.

\section{References}

Ackermann, M., Ajello, M., Albert, A., et al.: 2014, High-energy gamma-ray emission from solar flares: Summary of Fermi large area telescope detections and analysis of two M-class flares. Astrophys. J. 787, 15. DOI. ADS.

Ackermann, M., Allafort, A., Bardini, L., et al.: 2017, Fermi-LAT observations of high-energy behind-thelimb solar flares. Astrophys. J. 835, 219. DOI. ADS. 
Afanasiev, A., Aran, A., Vainio, R., Rouillard, A., Zucca, P., Lario, D., Barcewicz, S., Siipola, R., Pomoell, J., Sanahuja, B., Malandrakim, O.E.: 2018, Modelling of shock-accelerated gamma-ray events. In: Malandraki, O.E., Crosby, N.B. (eds.) Solar Particle Radiation Storms Forecasting and Analysis, Astrophys. Space Sci. Lib. Ser. 444, 157. DOI. ADS.

Akimov, V.V., Afanassyev, V.G., Belaousov, A.S., Blokhintsev, I.D., Kalinkin, L.F., Leikov, N.G., et al.: 1991, Observation of High Energy Gamma-rays from the Sun with the GAMMA-1 Telescope $(E>$ $30 \mathrm{MeV})$. In: 22nd International Cosmic Ray Conf. 3, 73. ADS.

Atwood, W.B., Abdo, A.A., Ackermann, M., Althouse, W., Anderson, B., Axelsson, M., et al.: 2009, The Large Area Telescope on the Fermi Gamma-Ray Space Telescope mission. Astrophys. J. 697, 1071. DOI. ADS.

Bougeret, J.-L., Kaiser, M.L., Kellogg, P.J., Manning, R., Goetz, L., Monson, S.J., Monge, N., Friel, L., Meetre, C.A., Perche, C., Sitruk, L., Hoang, S.: 1995, Waves: The radio and plasma wave investigation on the wind spacecraft. Space Sci. Rev. 71, 231. DOI. ADS.

Bougeret, J.-L., Goetz, K., Kaiser, M.L., Bale, S.D., Kellogg, P.J., Maksimovic, M., et al.: 2008, S/WAVES: The radio and plasma wave investigation on the STEREO mission. Space Sci. Rev. 136, 487. DOI. ADS.

Brueckner, G.E., Howard, R.A., Koomen, M.J., Korendyke, C.M., Michels, D.J., Moses, J.D., Socker, D.G., Dere, K.P., Lamy, P.L., Llebaria, A., Bout, M.V., Schwenn, R., Simnett, G.M., Bedford, D.K., Eyles, C.J.: 1995, The large angle spectroscopic coronagraph (LASCO). Solar Phys. 162, 357. DOI. ADS.

Bruno, A., Bazilevskaya, G.A., Boezio, M., Christian, E.R., de Nolfo, G.A., Martucci, M., et al.: 2018, Solar energetic particle events observed by the PAMELA mission. Astrophys. J. 862, 97. DOI. ADS.

Carley, E.P., Vilmer, N., Simões, P.J.A., Ó Fearraigh, B.: 2017, Estimation of a coronal mass ejection magnetic field strength using radio observations of gyrosynchrotron radiation. Astron. Astrophys. 608, A137. DOI. ADS.

Chertok, I.M., Belov, A.V., Grechnev, V.V.: 2015, A simple way to estimate the soft X-ray class of far-side solar flares observed with STEREO/EUVI. Solar Phys. 290, 1947. DOI. ADS.

Cliver, E.W., Kahler, S.W., Vestrand, W.T.: 1993, On the origin of gamma-RayEmission from the behind-thelimb flare on 29 September 1989. In: Leahy, D.A., Hicks, R.B., Venkatesan, D. (eds.) 23rd International Cosmic Ray Conf. 3, World Scientific, Singapore, 91. ADS.

Cohen, C.M.S., Mewaldt, R.A.: 2018, The ground-level enhancement event of September 2017 and other large solar energetic particle events of Cycle 24. Space Weather 16, 1616. DOI. ADS.

De Nolfo, G.A., Bruno, A., Ryan, J.M., Dalla, S., Giacalone, J., Richardson, I.G., Christian, E.R., Stochaj, S.J., Bazilevskaya, G.A., Boezio, M., Martucci, M., Mikhailov, V.V., Munini, R.: 2019a, Comparing long-duration gamma-ray flares and high-energy solar energetic particles. Astrophys. J. 879, 90. DOI. ADS.

De Nolfo, G.A., Bruno, A., Ryan, J.M., Dalla, S., Giacalone, J., Richardson, I.G., Christian, E.R.: 2019b, Long duration gamma-ray flares and high energy solar energetic particles: Is there a connection? In: 36th International Cosmic Ray Conf. 358, 1073. https://pos.sissa.it/358/1073/. ADS.

Dissauer, K., Veronig, A.M., Temmer, M., Podladchikova, T., Vanninathan, K.: 2018, On the detection of coronal dimmings and the extraction of their characteristic properties. Astrophys. J. 855, 137. DOI. ADS.

Domingo, V., Fleck, B., Poland, A.I.: 1995, The SOHO mission: An overview. Solar Phys. 162, 1. DOI. ADS.

Forrest, D.J., Vestrand, W.T., Chupp, E.L., Rieger, E., Cooper, J.F., Share, G.H.: 1985, Neutral pion production in solar flares. In: 19th International Cosmic Ray Conf. 4, 146. ADS.

Gonzalez, W.D., Clúa De Gonzalez, A.L., Dal Lago, A., Tsurutani, B.T., Arballo, J.K., Lakhina, G.S., Buti, B., Ho, G.M.: 1998, Magnetic cloud field intensities and solar wind velocities. Geophys. Res. Lett. 25, 963. DOI. ADS.

Gopalswamy, N.: 2006, Coronal mass ejections and Type II radio bursts. In: Gopalswamy, N., Mewaldt, R., Torsti, J. (eds.) Solar Eruptions and Energetic Particles, Geophysical Monograph Ser. 165, Am. Geophys. Union, Washington 207. DOI. ADS.

Gopalswamy, N.: 2009, Coronal mass ejections and space weather. In: Tsuda, T., Fujii, R., Shibata, K., Geller, M.A. (eds.) Climate and Weather of the Sun-Earth System (CAWSES): Selected Papers 2007 Kyoto Sympo., Terrapub, Tokyo, 77. http://www.terrapub.co.jp/onlineproceedings/ste/ CAWSES2007/index.html. ADS.

Gopalswamy, N.: 2011, Coronal mass ejections and solar radio emissions. In: Rucker, H.O., Kurth, W.S., Louarn, P., Fischer, G. (eds.) Planetary, Solar and Heliospheric Radio Emissions VII, Austrian Academy of Sciences Press, Vienna 325. ADS.

Gopalswamy, N., Mäkelä, P.: 2014, Latitudinal connectivity of ground level enhancement events. In: Hu, Q., Zank, G.P. (eds.) Coronal Heating to the Edge of the Heliosphere, ASP Conf. Ser. 484, 63. ADS.

Gopalswamy, N., Yashiro, S.: 2011, The strength and radial profile of the coronal magnetic field from the standoff distance of a coronal mass ejection-driven shock. Astrophys. J. 736, L11. DOI. ADS. 
Gopalswamy, N., Yashiro, S., Akiyama, S., Mäkelä, P., Xie, H., Kaiser, M.L., Howard, R., Bougeret, J.-L.: 2008, Coronal mass ejections, type II radio bursts, and solar energetic particle events in the SOHO era. Ann. Geophys. 26, 3033. DOI. ADS.

Gopalswamy, N., Yashiro, S., Michalek, G., Stenborg, G., Vourlidas, A., Freeland, S., Howard, R.: 2009a, The SOHO/LASCO CME catalog. Earth Moon Planets 104, 295. DOI. ADS.

Gopalswamy, N., Thompson, W.T., Davila, J.M., Kaiser, M.L., Yashiro, S., Mäkelä, P., Michalek, G., Bougeret, J.-L., Howard, R.A.: 2009b, Relation between type II bursts and CMEs inferred from STEREO observations. Solar Phys. 259, 227. DOI. ADS.

Gopalswamy, N., Xie, H., Akiyama, S., Yashiro, S., Usoskin, I.G., Davila, J.M.: 2013a, The first ground level enhancement event of solar cycle 24: Direct observation of shock formation and particle release heights. Astrophys. J. Lett. 765, L30. DOI. ADS.

Gopalswamy, N., Xie, H., Mäkelä, P., Yashiro, S., Akiyama, S., Uddin, W., et al.: 2013b, Height of shock formation in the solar corona inferred from observations of type II radio bursts and coronal mass ejections. Adv. Space Res. 51, 1981. DOI. ADS.

Gopalswamy, N., Mäkelä, P., Xie, H., Yashiro, S.: 2013c, Testing the empirical shock arrival model using quadrature observations. Space Weather 11, 661. DOI. ADS.

Gopalswamy, N., Mäkelä, P., Yashiro, S., Xie, H., Akiyama, S., Thakur, N.: 2015a, High-energy solar particle events in cycle 24. J. Phys. Conf. Ser. 642, 012012. DOI. ADS.

Gopalswamy, N., Yashiro, S., Xie, H., Akiyama, S., Mäkelä, P.: 2015b, Properties and geoeffectiveness of magnetic clouds during solar cycles 23 and 24. J. Geophys. Res. 120, 9221. DOI. ADS.

Gopalswamy, N., Akiyama, S., Mäkelä, P., Yashiro, S., Cairns, I.H.: 2016a, On the directivity of lowfrequency type IV radio bursts. In: URSI Asia-Pacific Radio Sci. Conf. (URSI AP-RASC), 1247. ADS.

Gopalswamy, N., Yashiro, S., Thakur, N., Mäkelä, P., Xie, H., Akiyama, S.: 2016b, The 2012 July 23 backside eruption: An extreme energetic particle event? Astrophys. J. 833, 216. DOI. ADS.

Gopalswamy, N., Mäkelä, P., Yashiro, S., Lara, A., Xie, H., Akiyama, S., MacDowall, R.J.: 2018a, Interplanetary type II radio bursts from wind/WAVES and sustained gamma-ray emission from Fermi/LAT: Evidence for shock source. Astrophys. J. Lett. 868, L19. DOI. ADS.

Gopalswamy, N., Mäkelä, P., Akiyama, S., Yashiro, S., Xie, H., Thakur, N.: 2018b, Sun-to-Earth propagation of the 2015 June 21 coronal mass ejection revealed by optical, EUV, and radio observations. J. Atmos. Solar-Terr. Phys. 179, 225. DOI. ADS.

Gopalswamy, N., Yashiro, S., Mäkelä, P., Xie, H., Akiyama, S., Monstein, C.: 2018c, Extreme kinematics of the 2017 September 10 solar eruption and the spectral characteristics of the associated energetic particles. Astrophys. J. 863, L39. DOI. ADS.

Gopalswamy, N., Akiyama, S., Yashiro, S., Xie, H.: 2018d, Coronal flux ropes and their interplanetary counterparts. J. Atmos. Solar-Terr. Phys. 180, 35. DOI. ADS.

Gopalswamy, N., Mäkelä, P., Yashiro, S., Lara, A., Akiyama, S., Xie, H.: 2019, On the shock source of sustained $\gamma$-ray emission from the Sun. J. Phys. Conf. Ser. 1332(1), 012004. DOI. ADS.

Grechnev, V., Kiselev, V.I., Kashapova, L.K., Kochanov, A.A., Zimovets, I.V., Uralov, A.M., Nizamov, B.A., Grigorieva, I.Y., Golovin, D.V., Litvak, M.L., Mitrofanov, I.G., Sanin, A.B.: 2018, Radio, hard X-ray, and gamma-ray emissions associated with a far-side solar event. Solar Phys. 293, 133. DOI. ADS.

Hess, P., Zhang, J.: 2014, Stereoscopic study of the kinematic evolution of a coronal mass ejection and its driven shock from the Sun to the Earth and the prediction of their arrival times. Astrophys. J. 792, 49. DOI. ADS.

Howard, R.A., Moses, J.D., Vourlidas, A., Newmark, J.S., Socker, D.G., Plunkett, S.P., et al.: 2008, Sun Earth Connection Coronal and Heliospheric Investigation (SECCHI). Space Sci. Rev. 136, 67. DOI. ADS.

Hudson, H.S.: 2018, The relationship between long-duration gamma-ray flares and solar cosmic rays. In: Foullon, C., Malandraki, O. (eds.) Space Weather of the Heliosphere: Processes and Forecasts. IAU Symp. 335, Univ. Press, Cambridge, Cambridge, 49. DOI. ADS.

Jin, M., Petrosian, V., Liu, W., Nitta, N.V., Omodei, N., Rubio da Costa, F., Effenberger, F., Li, G., PesceRollins, M., Allafort, A., Manchester, W.I.V.: 2018, Probing the puzzle of behind-the-limb $\gamma$-ray flares: Data-driven simulations of magnetic connectivity and CME-driven shock evolution. Astrophys. J. 867, 122. DOI. ADS.

Kahler, S.W., Cliver, E.W., Kazachenko, M.: 2018, Magnetic flux reconnection in flaring active regions with sustained gamma-ray emission. Astrophys. J. 868, 81. DOI. ADS.

Kahler, S.W., Hildner, E., Van Hollebeke, M.A.I.: 1978, Prompt solar proton events and coronal mass ejections. Solar Phys. 57, 429. DOI. ADS.

Kaiser, M.L., Kucera, T.A., Davila, J.M., St. Cyr, O.C., Guhathakurta, M., Christian, E.: 2008, The STEREO mission: An introduction. Space Sci. Rev. 136, 5. DOI. ADS.

Kanbach, G., Bertsch, D.L., Fichtel, C.E., Hartman, R.C., Hunter, S.D., Kniffen, D.A., Kwok, P.W., Lin, Y.C., Mattox, J.R., Mayer-Hasselwander, H.A.: 1993, Detection of a long-duration solar gamma-ray flare on June 11, 1991 with EGRET on COMPTON-GRO. Astron. Astophys. Suppl. Ser. 97, 349. ADS. 
Klein, K.-L., Tziotziou, K., Zucca, P., Valtonen, E., Vilmer, N., Malandraki, O.E., Hamadache, C., Heber, B., Kiener, J.: 2018, X-ray, radio and SEP observations of relativistic gamma-ray events. Astrophys. Space Sci. Libr. 444, 133. DOI. ADS.

Kocharov, L.G., Laitinen, T., Vainio, R., Afanasiev, A., Mursula, K., Ryan, J.M.: 2015, Solar interacting protons versus interplanetary protons in the core plus halo model of diffusive shock acceleration and stochastic re-acceleration. Astrophys. J. 806, 80. DOI. ADS.

Mäkelä, P., Gopalswamy, N., Akiyama, S., Xie, H., Yashiro, S.: 2015, Estimating the height of CMEs associated with a major SEP event at the onset of the metric type II radio burst during solar cycles 23 and 24. Astrophys. J. 806, 13. DOI. ADS.

Mann, G., Klassen, A., Classen, H.T., Aurass, H., Scholz, D., MacDowall, R.J., Stone, R.G.: 1996, Catalogue of solar type II radio bursts observed from September 1990 to December 1993 and their statistical analysis. Astron. Astrophys. 119, 489. ADS.

Mewaldt, R.A., Cohen, C.M.S., Mason, G.M., Haggerty, D.K., Looper, M.D., Vourlidas, A., Desai, M.I., Giacalone, J., Labrador, A.W., Leske, R.A., Mazur, J.E.: 2005, How efficient are coronal mass ejections at accelerating solar energetic particles? In: Fleck, B., Zurbuchen, T.H., Lacoste, H. (eds.) Solar Wind 11/SOHO 16, Connecting Sun and Heliosphere (ESA SP) 592, 67. ADS.

Murphy, R.J., Dermer, C.D., Ramaty, R.: 1987, High-energy processes in solar flares. Astrophys. J. 63, 721. DOI. ADS.

Nitta, N.V., Aschwanden, M.J., Boerner, P.F., Freeland, S.L., Lemen, J.R., Wuelser, J.-P.: 2013, Soft X-ray fluxes of major flares far behind the limb as estimated using STEREO EUV images. Solar Phys. 288, 241. DOI. ADS.

Olmedo, O., Gopalswamy, N., Xie, H., Yashiro, S., Mäkelä, P.A., Akiyama, S., St. Cyr, O.C., Vourlidas, A.: 2013, Forward fitting of a coronal shock front to a spheroid. In: American Geophys. Uni. Fall Meeting 2013 SH13A-2033. ADS.

Pesce-Rollins, M., Omodei, N., Petrosian, V., Liu, W., Rubio da Costa, F., Allafort, A., Chen, Q.: 2015a, First detection of \&gt100 MeV gamma rays associated with a behind-the-limb solar flare. Astrophys. J. Lett. 805, L15. DOI. ADS.

Pesce-Rollins, M., Omodei, N., Petrosian, V., Liu, W., Rubio da Costa, F., Allafort, A. (FermiLAT Collaboration): 2015b, Fermi Large Area Telescope observations of high-energy gammaray emission from behind-the-limb solar flares. In: 34th International Cosmic Ray Conf., 128. https://pos.sissa.it/cgi-bin/reader/conf.cgi?confid=236. ADS.

Plotnikov, I., Rouillard, A.P., Share, G.H.: 2017, The magnetic connectivity of coronal shocks from behind the-limb flares to the visible solar surface during $\gamma$-ray events. Astron. Astrophys. 608, A43. DOI. ADS.

Ramaty, R., Murphy, R.J., Dermer, C.D.: 1987, On the origin of the pion-decay radiation in the 1982 June 3 solar flare. Astrophys. J. Lett. 316, L41. DOI. ADS.

Reames, D.V.: 1999, Particle acceleration at the Sun and in the heliosphere. Space Sci. Rev. 90, 413. DOI. ADS.

Ryan, J.M.: 2000, Long-duration solar gamma-ray flares. Space Sci. Rev. 93, 581. DOI. ADS.

Share, G.H., Murphy, R.J., White, S.M., Tolbert, A.K., Dennis, B.R., Schwartz, R.A., Smart, D.F., Shea, M.A.: 2018, Characteristics of late-phase $>100 \mathrm{MeV}$ gamma-ray emission in solar eruptive events. Astrophys. J. 869, 182. DOI. ADS.

Talon, R., Trottet, G., Vilmer, N., Barat, C., Dezalay, J.-P., Sunyaev, R., Terekhov, O., Kuznetsov, A.: 1993, Hard X-ray and $\gamma$-ray observations of solar flares with the Phebus experiment. Solar Phys. 147, 137. DOI. ADS.

Thernisien, A.: 2011, Implementation of the graduated cylindrical shell model for the three-dimensional reconstruction of coronal mass ejections. Astrophys. J. Supp. Ser. 194, 33. DOI. ADS.

Tousey, R.: 1973, The solar corona. In: Rycroft, M.J., Runcorn, S.K. (eds.) Space Research XIII, Akademie Verlag, Berlin, 713. ADS.

Vainio, R.: 2009, Particle acceleration and turbulence transport in heliospheric plasmas. In: Universal Heliophysical Processes, Proc. of the International Astronomical Union Symposium 257, 413. DOI. ADS.

Vestrand, W.T., Forrest, D.J.: 1993, Evidence for a spatially extended component of gamma rays from solar flares. Astrophys. J. 409, L69. DOI. ADS.

Vilmer, N., MacKinnon, A.L., Trottet, G., Barat, C.: 2003, High energy particles accelerated during the large solar flare of 1990 May 24: X/ $\gamma$-ray observations. Astron. Astrophys. 412, 865. DOI. ADS.

von Rosenvinge, T.T., Reames, D.V., Baker, R., Hawk, J., Nolan, J.T., Ryan, L., Shuman, S., Wortman, K.A., Mewaldt, R.A., Cummings, A.C., Cook, W.R., Labrador, A.W., Leske, R.A., Wiedenbeck, M.E.: 2008, The High Energy Telescope for STEREO. Space Sci. Rev. 136, 391. DOI. ADS.

Webb, D.F., Cliver, E.W., Crooker, N.U., St. Cyr, O.C., Thompson, B.J.: 2000, Relationship of halo coronal mass ejections, magnetic clouds, and magnetic storms. J. Geophys. Res. 105, 7491. DOI. ADS.

Winter, L.M., Bernstein, V., Omodei, V., Pesce-Rollins, M.: 2018, A statistical study to determine the origin of long-duration gamma-ray flares. Astrophys. J. 864, 39. DOI. ADS. 
Wolff, M., Tautz, R.C.: 2015, Cosmic-ray acceleration at collisionless astrophysical shocks using MonteCarlo simulations. Astron. Astrophys. 580, A58. DOI. ADS.

Xie, H., Mäkelä, P., St. Cyr, O.C., Gopalswamy, N.: 2017, Comparison of the coronal mass ejection shock acceleration of three widespread SEP events during solar cycle 24. J. Geophys. Res. 122, 7021. DOI. ADS.

Yashiro, S., Gopalswamy, N., Michalek, G., St. Cyr, O.C., Plunkett, S.P., Rich, N.B., Howard, R.A.: 2004, A catalog of white light coronal mass ejections observed by the SOHO spacecraft. J. Geophys. Res. 109, A07105. DOI. ADS. 